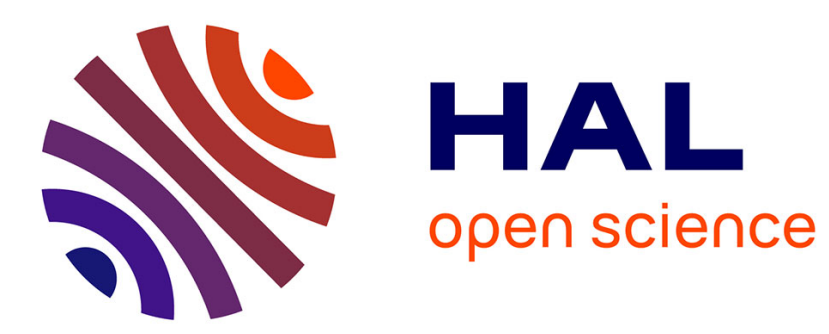

\title{
Numerical treatment of two-dimensional interfaces for acoustic and elastic waves
}

\author{
Bruno Lombard, Joël Piraux
}

\section{To cite this version:}

Bruno Lombard, Joël Piraux. Numerical treatment of two-dimensional interfaces for acoustic and elastic waves. Journal of Computational Physics, 2004, 195 (1), pp.90-116. 10.1016/j.jcp.2003.09.024 . hal-00004813

\section{HAL Id: hal-00004813 \\ https://hal.science/hal-00004813}

Submitted on 29 Apr 2005

HAL is a multi-disciplinary open access archive for the deposit and dissemination of scientific research documents, whether they are published or not. The documents may come from teaching and research institutions in France or abroad, or from public or private research centers.
L'archive ouverte pluridisciplinaire HAL, est destinée au dépôt et à la diffusion de documents scientifiques de niveau recherche, publiés ou non, émanant des établissements d'enseignement et de recherche français ou étrangers, des laboratoires publics ou privés. 


\title{
Numerical treatment of two-dimensional interfaces for acoustic and elastic waves.
}

\author{
Bruno Lombard, Joël Piraux \\ Laboratoire de Mécanique et d'Acoustique, 31 chemin Joseph Aiguier, 13402 \\ Marseille, France
}

\begin{abstract}
We present a numerical method to take into account 2D arbitrary-shaped interfaces in classical finite-difference schemes, on a uniform Cartesian grid. This work extends the "Explicit Simplified Interface Method" (ESIM), previously proposed in 1D (2001, J. Comput. Phys. 168, pp. 227-248). The physical problem under study concerns the linear hyperbolic systems of acoustics and elastodynamics, with stationary interfaces. Our method maintains, near the interfaces, properties of the schemes in homogeneous medium, such as the order of accuracy and the stability limit. Moreover, it enforces the numerical solution to satisfy the exact interface conditions. Lastly, it provides subcell geometrical features of the interface inside the meshing. The ESIM can be coupled automatically with a wide class of numerical schemes (Lax-Wendroff, flux-limiter schemes,...) for a negligible additional computational cost. Throughout the paper, we focus on the challenging case of an interface between a fluid and an elastic solid. In numerical experiments, we provide comparisons between numerical solutions and original analytic solutions, showing the efficiency of the method.
\end{abstract}

Key words: Acoustics and Elastodynamics, Discontinuous Coefficients, Jump Conditions, Interface Methods, Wave Propagation Algorithm, Singular Value Decomposition, Hyperbolic Systems.

1991 MSC: 35L40, 65M06

\section{Introduction}

Consider two-dimensional perfect fluids and elastic solids, with discontinuous physical properties across arbitrary-shaped interfaces. We want to simulate

Email addresses: lombard@lma.cnrs-mrs.fr (Bruno Lombard), piraux@lma.cnrs-mrs.fr (Joël Piraux). 
the propagation of acoustic waves and elastic waves in such media. To do so, we use classical finite-difference or finite-volume schemes on a uniform Cartesian grid. Without an efficient numerical treatment of the interface, we can neither expect high-quality simulations nor have any confidence in the results. It follows from three reasons. First, the stair-step representation of arbitrary-shaped interfaces introduces spurious diffractions [4]. Second, the non-smoothness of the solution across the interfaces reduces the order of convergence [28], and numerical instabilities can occur even for low contrasts of physical parameters. Third, the jump conditions and the boundary conditions are not incorporated in the schemes, so that the conversion, refraction, and diffraction wave phenomenons are not correctly described [18].

(a)

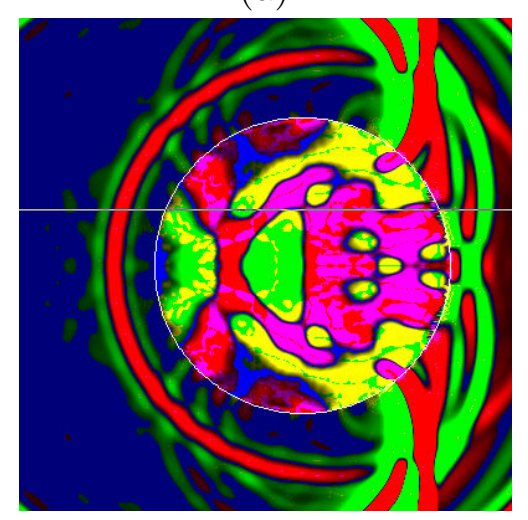

(b)

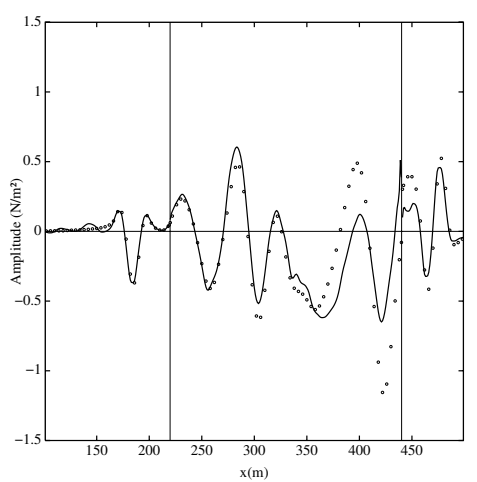

Fig. 1. Plane wave on a circular interface, without an interface treatment (green-red: P-waves, magenta-yellow: SV-waves; exact solution: points, numerical solution: solid line).

To see what happens when the interfaces are not taken into account properly, we show in figure 1 a simulation of a plane wave in a fluid, interacting with a circular elastic medium. The parameters and the results are detailed in section 4.3. As often done in practice, the fluid is considered as a solid with an almost-zero celerity of transverse waves. A classical scheme is used everywhere, without any special care near the interfaces. The analytical solution is denoted by points; the numerical solution (computed by the Wave Propagation Algorithm of LeVeque [14]) is denoted by a solid line. The agreement between analytical and numerical values is very bad, and one can observe unphysical waves.

The goal of our paper is to propose a numerical method to avoid these problems induced by the interfaces. Our method can be coupled with a wide class of numerical schemes, maintaining some properties of those schemes in homogeneous medium. It is instructive to compare the figure 1 with the figure 6 $(i=5)$, where our method is applied.

Our strategy belongs to the family of the interface methods, whose basic prin- 
ciple is the following. Consider an interface immerged in a regular Cartesian grid. One uses a classical scheme far from the interfaces, like in homogeneous medium. Another scheme is used near the interfaces (more precisely, at the grid points where the stencil crosses an interface). This new scheme is built from the jump conditions to ensure the same order of accuracy as the first scheme.

To our knowledge, the common ancestor of the interface methods is the "Immersed Boundary Method" (IBM), developed by Peskin in 1977 [21]. Its purpose was to describe an elastic membrane and discrete forces in a Stokes flow. The IBM is first-order accurate: to improve the accuracy near the interfaces, LeVeque and his collaborators proposed the "Immersed Interface Method" (IIM). Let us mention some works based on the IIM: Li in 1994 for elliptic equations [16] and in 1997 for Stokes flow [17], Zhang in 1996 for hyperbolic systems of acoustics and elastodynamics [27,28], Wiegmann in 1998 for nonlinear parabolic equations [25], Calhoun in 2000 for advection-diffusion with obstacles [3], Lee in 2002 for Navier-Stokes equations with interfaces [11,12]. An alternative of the IIM was proposed for elliptic equations by Wiegmann and Bube in 1998: the "Explicit Jump Immersed Interface Method" (EJIIM) $[26]$.

Despite its qualities, the IIM suffers from some drawbacks for an efficient application to acoustics and elastodynamics. Even if we use sophisticated schemes far from the interfaces (such as the Wave Propagation Algorithm) to avoid the numerical dispersion, the IIM introduces some unphysical dispersion near the interfaces. The coupling of the IIM with high-order and sophisticated schemes is non-trivial. Lastly, numerical instabilities are observed, even for moderate contrasts of the physical parameters [18].

Those drawbacks has lead us to propose a new interface method in $1 \mathrm{D}$, the "Explicit Simplified Interface Method" (ESIM) [22]. The coupling of the ESIM with any scheme is so intimate that the numerical properties are the same at any point, far or near the interfaces. Moreover, this coupling is automatic and independant of the scheme. The key idea of the ESIM is simple: instead of modifying explicitly a scheme near the interfaces, we modify some numerical values used for time-stepping near the interfaces. These modified values are deduced from smooth extensions of the solution on both sides of the interface. These smooth extensions are based on the jump conditions satisfied by the exact solution at the interface.

The underlying philosophy of the ESIM is quite similar to the "Ghost Fluid Method" (GFM) proposed by Fedkiw and al. [5] for multicomponent Euler flows. However, we believe that our method is more accurate than the GFM in the context of linear equations and stationary interfaces. Indeed, we take into account precisely the geometrical features of the interface, and we can 
reach arbitrary-high orders of precision.

The present paper extends the ESIM to two-dimensional configurations. Even if the key idea is the same as in the one-dimensional case, many new ideas are introduced. First, we take into account complicated geometries, and different media on both sides of the interfaces (e.g. fluid and solid). Second, we consider various interface conditions: jump conditions, boundary conditions, and compatibility conditions. Third, in many cases, the number of interface conditions is lower than the number of components of the solution: this underdetermination complicates the procedure.

The ESIM has been tested in many two-dimensional cases: fluid-fluid, solidsolid in perfect or imperfect contact, fluid-solid [18]. The numerical treatment proposed further can be applied for all these cases. However, we will mainly focus on the fluid-solid case, especially in numerical experiments. This configuration is particularly challenging because of its difficulties and the applications, to cite a few: the wave propagation in water and solid sediments in underwater acoustics, and in the human bone in biomechanics. To our knowledge, the fluid-solid interface has not been treated by other interface methods, because of the underdetermination of the interface conditions. Up to now, the fictitious domain method [4] do not treat this configuration. The spectral element method applied in [10] treats the fluid-solid interface, but it requires that the grid points coincide with the interface. Lastly, alternative methods based on the averaging of the coefficients near the interfaces $[6,24]$ are, at best, first-order accurate.

The paper is organized as follows. The section 2 contains the basic tools for the study. The first-order hyperbolic systems of conservation laws for acoustics and elastodynamics are recalled. We write the interface conditions, and we shortly recall the numerical schemes used far from the interfaces. The section 3 describes the algorithm of the ESIM. In section 4, we propose numerical experiments for fluid-solid interfaces, and comparisons with analytical solutions in three configurations: a plane interface, a circular interface, a circular elastic shell immerged in a fluid. Finally, some conclusions are drawn in section 5. 


\section{General framework}

\subsection{Conservation laws}

We are concerned with the propagation of small perturbations in initially motionless fluids and isotropic elastic solids. The physical properties are supposed piecewise constant, and they are discontinuous across stationary interfaces. To fix the ideas, consider two media $\Omega_{i}(i=1,2)$ separated by an interface $\Gamma$ (figure 2). We use a parametric representation of $\Gamma:(x(\tau), y(\tau))$. The tangential vector $\boldsymbol{t}$ and the normal vector $\boldsymbol{n}$ are given by

$$
\boldsymbol{t}=\left(\begin{array}{c}
x^{\prime} \\
y^{\prime}
\end{array}\right), \quad \boldsymbol{n}=\left(\begin{array}{c}
-y^{\prime} \\
x^{\prime}
\end{array}\right),
$$

where we use the notation $x^{\prime}=\frac{d x}{d \tau}$ and $y^{\prime}=\frac{d y}{d \tau}$. The interface $\Gamma$ is supposed sufficiently smooth: $x(\tau), y(\tau)$ and their spatial derivatives (up to a given order) are continuous all along $\Gamma$.

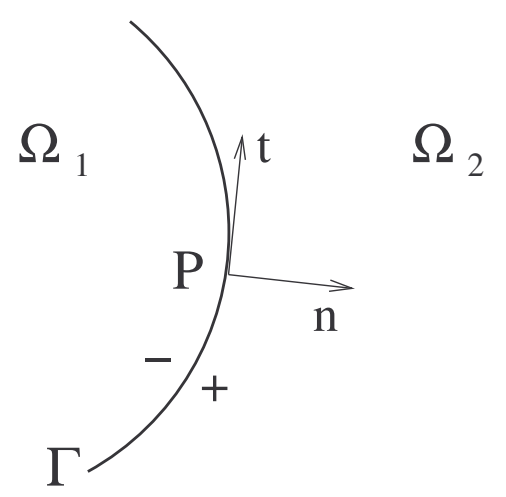

Fig. 2. Two media $\Omega_{1}$ and $\Omega_{2}$ separated by an interface $\Gamma$.

If $\Omega_{i}$ is a fluid, the physical parameters are the density $\rho$ and the sound speed $c$. The unknowns are the two components of the acoustic velocity $\boldsymbol{v}\left(v_{1}, v_{2}\right)$, and the acoustic pressure $p$. Then, the acoustic solution is denoted by

$$
\boldsymbol{U}={ }^{T}\left(v_{1}, v_{2}, p\right)
$$

If $\Omega_{i}$ is a solid, the physical parameters are the density $\rho$ and the elastic speeds of $\mathrm{P}$-waves and S-waves $c_{p}$ and $c_{s}$, linked to the Lamé coefficients $\lambda, \mu$ by

$$
c_{p}=\sqrt{\frac{\lambda+2 \mu}{\rho}}, \quad c_{s}=\sqrt{\frac{\mu}{\rho}} .
$$


The unknowns are the two components of the elastic velocity $\boldsymbol{v}\left(v_{1}, v_{2}\right)$, and the three independant components of the elastic stress tensor $\boldsymbol{\sigma}\left(\sigma_{11}, \sigma_{12}, \sigma_{22}\right)$. Then, the elastic solution is denoted by

$$
\boldsymbol{U}={ }^{T}\left(v_{1}, v_{2}, \sigma_{11}, \sigma_{12}, \sigma_{22}\right)
$$

In both cases, the linearization of the mechanic equations on medium $\Omega_{i}$ and outside $\Gamma$ leads to a first-order linear hyperbolic system

$$
\frac{\partial}{\partial t} \boldsymbol{U}+\boldsymbol{A}_{i} \frac{\partial}{\partial x} \boldsymbol{U}+\boldsymbol{B}_{i} \frac{\partial}{\partial y} \boldsymbol{U}=\mathbf{0}
$$

where the matrices $\boldsymbol{A}_{i}$ and $\boldsymbol{B}_{i}$ depend on the physical parameters (for the sake of clarity, we omit the indices $i$ in these parameters). In the fluid case, the $3 \times 3$ matrices $\boldsymbol{A}_{i}$ and $\boldsymbol{B}_{i}$ are

$$
\boldsymbol{A}_{i}=\left(\begin{array}{ccc}
0 & 0 & \frac{1}{\rho} \\
0 & 0 & 0 \\
\rho c^{2} & 0 & 0
\end{array}\right), \quad \boldsymbol{B}_{i}=\left(\begin{array}{ccc}
0 & 0 & 0 \\
0 & 0 & \frac{1}{\rho} \\
0 & \rho c^{2} & 0
\end{array}\right) .
$$

In the solid case, the $5 \times 5$ matrices $\boldsymbol{A}_{i}$ and $\boldsymbol{B}_{i}$ are

$$
\boldsymbol{A}_{i}=-\left(\begin{array}{ccccc}
0 & 0 & \frac{1}{\rho} & 0 & 0 \\
0 & 0 & 0 & \frac{1}{\rho} & 0 \\
\rho c_{p}^{2} & 0 & 0 & 0 & 0 \\
0 & \rho c_{s}^{2} & 0 & 0 & 0 \\
\rho\left(c_{p}^{2}-2 c_{s}^{2}\right) & 0 & 0 & 0 & 0
\end{array}\right),
$$




$$
\boldsymbol{B}_{i}=-\left(\begin{array}{ccccc}
0 & 0 & 0 & \frac{1}{\rho} & 0 \\
0 & 0 & 0 & 0 & \frac{1}{\rho} \\
0 & \rho\left(c_{p}^{2}-2 c_{s}^{2}\right) & 0 & 0 & 0 \\
\rho c_{s}^{2} & 0 & 0 & 0 & 0 \\
0 & \rho c_{p}^{2} & 0 & 0 & 0
\end{array}\right) .
$$

\subsection{The interface conditions}

Consider a point $P$ of $\Gamma$ (figure 2). On both sides of $P$, the limit values of the solution $\boldsymbol{U}(x, y, t)$ and of its spatial derivatives up to the $k$-th order are denoted by

$$
\boldsymbol{U}_{i}^{k}=\lim _{M \rightarrow P, M \in \Omega_{i}}\left(\boldsymbol{U}, \frac{\partial}{\partial x} \boldsymbol{U}, \frac{\partial}{\partial y} \boldsymbol{U}, \ldots, \frac{\partial^{\alpha}}{\partial x^{\alpha-\beta} \partial y^{\beta}} \boldsymbol{U}, \ldots, \frac{\partial^{k}}{\partial y^{k}} \boldsymbol{U}\right),
$$

with $\alpha=0, \ldots, k$ and $\beta=0, \ldots, \alpha$. The vector $\boldsymbol{U}_{i}^{k}$ has $3(k+1)(k+2) / 2$ components if $\Omega_{i}$ is fluid, and $5(k+1)(k+2) / 2$ components if $\Omega_{i}$ is solid.

To well-define the wave propagation problem, one must give the jump conditions and the boundary conditions satisfied by the solution $\boldsymbol{U}$ along $\Gamma$. The classical interface conditions are written abstractly

$$
\begin{aligned}
& \boldsymbol{C}_{1}^{0} \boldsymbol{U}_{1}^{0}=\boldsymbol{C}_{2}^{0} \boldsymbol{U}_{2}^{0}, \\
& \boldsymbol{L}_{1}^{0} \boldsymbol{U}_{1}^{0}=\mathbf{0} \\
& \boldsymbol{L}_{2}^{0} \boldsymbol{U}_{2}^{0}=\mathbf{0}
\end{aligned}
$$

The first expression in (9) is a jump condition, the other are boundary conditions. The matrices $\boldsymbol{C}_{i}^{0}$ and $\boldsymbol{L}_{i}^{0}(i=1,2)$ depend on the parameter $\tau$, but they are independant of $t$. More sophisticated interface conditions could also be investigated, but they are beyond the scope of the present paper; see e.g. 
[19] for conditions describing imperfect contacts between solids. Unlike other authors who work in a local system of coordinates (see e.g. [28]), we work directly in the $(x, y)$ coordinates.

As an example, consider the fluid-solid case, where $\Omega_{1}$ is the fluid and $\Omega_{2}$ is the solid. Since the fluid is perfect, the normal velocity and the normal stresses are continuous, hence

$$
[\boldsymbol{v} \cdot \boldsymbol{n}]=0, \quad-p \boldsymbol{n}=\boldsymbol{\sigma} \boldsymbol{n}
$$

where [] denotes the jump across $\Gamma$, from $\Omega_{1}$ to $\Omega_{2}$. From (1) and (10), we recover (9) by setting

$$
\begin{aligned}
& \boldsymbol{C}_{1}^{0}=\left(\begin{array}{ccc}
-y^{\prime} & x^{\prime} & 0 \\
0 & 0 & x^{\prime 2}+y^{\prime 2}
\end{array}\right), \\
& \boldsymbol{C}_{2}^{0}=\left(\begin{array}{ccccc}
-y^{\prime} & x^{\prime} & 0 & 0 & 0 \\
0 & 0 & y^{\prime 2} & -2 x^{\prime} y^{\prime} & x^{\prime 2}
\end{array}\right), \\
& \boldsymbol{L}_{1}^{0}=\left(\begin{array}{lll}
0 & 0 & 0
\end{array}\right) \\
& \boldsymbol{L}_{2}^{0}=\left(\begin{array}{lllll}
0 & 0 & x^{\prime} y^{\prime} & y^{\prime 2}-x^{\prime 2} & -x^{\prime} y^{\prime}
\end{array}\right) .
\end{aligned}
$$

From the equation (10), we can seek to express the solution on the fluid side (2) in terms of the solution on the solid side (4), or inversely. In both cases, there are not enough equations, as shown now. In the first case, three unknowns must be determined; to do so, we deduce from (10) one equation for $p$ and only one equation for $v_{1}$ and $v_{2}$. In the second case, five unknowns must be determined; to do so, we have only one equation for $v_{1}$ and $v_{2}$, and two equations (one boundary condition and one jump condition) for $\sigma_{11}, \sigma_{12}$, and $\sigma_{22}$. This simple remark about the underdetermination of interface conditions leads to a rather complicated procedure explained in section 3.3. 


\subsection{Numerical schemes far from interfaces}

We define a time step $\Delta t$ and a uniform Cartesian grid with spatial meshes $\Delta x=\Delta y$. The approximation of $\boldsymbol{U}\left(x_{i}=i \Delta x, y_{j}=j \Delta y, t_{n}=n \Delta t\right)$ is denoted by $\boldsymbol{U}_{i, j}^{n}$. To integrate (5), one can use two-step finite-difference or finite-volume schemes, written symbolically

$$
\boldsymbol{U}_{i, j}^{n+1}=\boldsymbol{H}\left(\boldsymbol{U}_{i+\alpha, j+\beta}^{n}\right),
$$

where $\boldsymbol{H}$ is a discrete operator, and $\alpha, \beta$ depend on the chosen scheme. See $[9,13]$ for a survey of the huge literature dedicated to this subject.

For numerical experiments performed in section 4, we use second-order schemes: the Lax-Wendroff scheme (for its simplicity) and the Wave Propagation Algorithm (WPALG) of LeVeque [14]. This last 21-point truly multidimensional finite-volume scheme reduces the numerical anisotropy induced by the Cartesian grid. Moreover, the numerical dispersion is avoided via flux limiters. Lastly, it is stable up to $\mathrm{CFL}=1$.

The time-stepping (12) is applied at the regular points, that is at the grid points where the stencil belongs only to one medium. The aim of the present paper is to detail the time-stepping at the irregular points (not regular points), that is at the grid points where the stencil crosses the interface. Unlike 1D cases, no theoretical expression of the irregular points is available, because of the arbitrary shapes of the interfaces.

\section{The Explicit Simplified Interface Method}

The technical aspects of the algorithm are now detailed, followed by some remarks.

\subsection{Derivation of the interface conditions}

For further use, we need the jump conditions and the boundary conditions to be satisfied by $\boldsymbol{U}_{i}^{k}(i=1,2, k \geq 1)$. To do so, we differentiate (9) in terms of $t$ and $\tau$, and we use the conservation law (5).

To illustrate the procedure, consider the second equation of (9): $\boldsymbol{L}_{1}^{0} \boldsymbol{U}_{1}^{0}=\mathbf{0}$. First, we differentiate it in terms of $t$. Since $\boldsymbol{L}_{1}^{0}$ does not depend on $t$ ( $\Gamma$ is a 
stationary interface), we get

$$
\boldsymbol{L}_{1}^{0} \frac{\partial}{\partial t} \boldsymbol{U}_{1}^{0}=\mathbf{0}
$$

Then, the time derivative in (13) is replaced by spatial derivatives via the conservation law (5)

$$
-\boldsymbol{L}_{1}^{0} \boldsymbol{A}_{1} \frac{\partial}{\partial x} \boldsymbol{U}_{1}^{0}-\boldsymbol{L}_{1}^{0} \boldsymbol{B}_{1} \frac{\partial}{\partial y} \boldsymbol{U}_{1}^{0}=\mathbf{0} .
$$

Second, we differentiate $\boldsymbol{L}_{1}^{0} \boldsymbol{U}_{1}^{0}=\mathbf{0}$ in terms of $\tau$, leading to

$$
\left(\frac{d}{d \tau} \boldsymbol{L}_{1}^{0}\right) \boldsymbol{U}_{1}^{0}+\boldsymbol{L}_{1}^{0} \frac{\partial}{\partial \tau} \boldsymbol{U}_{1}^{0}=\mathbf{0} .
$$

Since $\boldsymbol{U}_{1}^{0}$ depends on $x(\tau), y(\tau)$, the chain-rule gives

$$
\left(\frac{d}{d \tau} \boldsymbol{L}_{1}^{0}\right) \boldsymbol{U}_{1}^{0}+\boldsymbol{L}_{1}^{0}\left(x^{\prime} \frac{\partial}{\partial x} \boldsymbol{U}_{1}^{0}+y^{\prime} \frac{\partial}{\partial y} \boldsymbol{U}_{1}^{0}\right) .
$$

We define the matrix-block

$$
\boldsymbol{L}_{1}^{1}=\left(\begin{array}{ccc}
\mathbf{0} & -\boldsymbol{L}_{1}^{0} \boldsymbol{A}_{1}-\boldsymbol{L}_{1}^{0} \boldsymbol{B}_{1} \\
\frac{d}{d \tau} \boldsymbol{L}_{1}^{0} & x^{\prime} \boldsymbol{L}_{1}^{0} & y^{\prime} \boldsymbol{L}_{1}^{0}
\end{array}\right) .
$$

Then, from (8), (14), and (16), we deduce

$$
\boldsymbol{L}_{1}^{1} \boldsymbol{U}_{1}^{1}=\mathbf{0}
$$

By iterating a similar procedure $k$ times, one can find matrices $\boldsymbol{C}_{i}^{k}$ and $\boldsymbol{L}_{i}^{k}$ $(i=1,2, k \geq 1)$ such that

$$
\begin{aligned}
& \boldsymbol{C}_{1}^{k} \boldsymbol{U}_{1}^{k}=\boldsymbol{C}_{2}^{k} \boldsymbol{U}_{2}^{k}, \\
& \boldsymbol{L}_{1}^{k} \boldsymbol{U}_{1}^{k}=\mathbf{0} \\
& \boldsymbol{L}_{2}^{k} \boldsymbol{U}_{2}^{k}=\mathbf{0}
\end{aligned}
$$

This leads to tedious calculations for high values of $k$; note that this task can be done automatically with formal calculus, as done in our own programs. 


\subsection{The compatibility conditions}

Some components of the spatial derivatives of $\boldsymbol{U}$ are not independant. They are linked together by compatibility conditions, useful to reduce the number of components in (19).

If $\Omega_{i}$ is a fluid, the vorticity is null outside $\Gamma$, hence

$$
\frac{\partial v_{1}}{\partial y}-\frac{\partial v_{2}}{\partial x}=0
$$

Differentiating (20) $(k-1)$-times in terms of $x$ and $y$ leads to

$$
\frac{\partial^{k} v_{1}}{\partial x^{k-j-1} \partial y^{j+1}}-\frac{\partial^{k} v_{2}}{\partial x^{k-j} \partial y^{j}}=0, \quad k \geq 1, j=0, \ldots, k-1 .
$$

If $\Omega_{i}$ is a solid, we set

$$
\alpha_{1}=\frac{c_{p}^{2}}{4\left(c_{p}^{2}-c_{s}^{2}\right)}, \quad \alpha_{2}=\frac{2 c_{s}^{2}-c_{p}^{2}}{4\left(c_{p}^{2}-c_{s}^{2}\right)} .
$$

A necessary and sufficient condition for the strain tensor $\boldsymbol{\sigma}$ to be symetrical is given by [8]

$$
\alpha_{2} \frac{\partial^{2} \sigma_{11}}{\partial x^{2}}+\alpha_{1} \frac{\partial^{2} \sigma_{22}}{\partial x^{2}}-\frac{\partial^{2} \sigma_{12}}{\partial x \partial y}+\alpha_{1} \frac{\partial^{2} \sigma_{11}}{\partial y^{2}}+\alpha_{2} \frac{\partial^{2} \sigma_{22}}{\partial y^{2}}=0 .
$$

Differentiating (23) $(k-2)$-times in terms of $x$ and $y$ leads to

$$
\begin{aligned}
& \alpha_{2} \frac{\partial^{k} \sigma_{11}}{\partial x^{k-j} \partial y^{j}}+\alpha_{1} \frac{\partial^{k} \sigma_{22}}{\partial x^{k-j} \partial y^{j}}-\frac{\partial^{k} \sigma_{12}}{\partial x^{k-j-1} \partial y^{j+1}} \\
& +\alpha_{1} \frac{\partial^{k} \sigma_{11}}{\partial x^{k-j-2} \partial y^{j+2}}+\alpha_{2} \frac{\partial^{k} \sigma_{22}}{\partial x^{k-j-2} \partial y^{j+2}}=0, \quad k \geq 2, j=0, \ldots, k-2 .
\end{aligned}
$$

The conditions (21) and (24) are satisfied at each point of $\Omega_{i}(i=1,2)$, especially at $P^{+}$and $P^{-}$(see figure 2 ). Hence, we can use the compatibility conditions (21) and (24) to reduce the number of independant components of $\boldsymbol{U}_{i}^{k}$. So, we write

$$
\boldsymbol{U}_{i}^{k}=\boldsymbol{G}_{i}^{k} \boldsymbol{V}_{i}^{k}, \quad i=1,2 .
$$

The vector $\boldsymbol{V}_{i}^{k}$ of independant variables has $(k+1)(k+3)$ components if $\Omega_{i}$ is fluid, or $2 k^{2}+8 k+5$ components if $\Omega_{i}$ is solid. The rectangular matrix $\boldsymbol{G}_{i}^{k}$ is detailed in Appendix A, both in the fluid case and in the solid case. 


\subsection{Some work on the interface conditions}

For further use in the ESIM, we need to express $\boldsymbol{U}_{2}^{k}$ in terms of $\boldsymbol{U}_{1}^{k}$ (and vice-versa). To do so, we use the interface conditions (19) and the relation (25) deduced from the compatibility conditions. Since the systems deduced from all these conditions are underdetermined, the solution is not unique. To find the full span of solutions, our strategy is based on Singular Value Decompositions (SVD). Technical details can be found in Appendix A.

Inserting (25) in the boundary conditions of (19) leads to a minimal set of independant components $\boldsymbol{W}_{i}^{k}$

$$
\boldsymbol{L}_{i}^{k} \boldsymbol{G}_{i}^{k} \boldsymbol{V}_{i}^{k}=\mathbf{0} \quad \Rightarrow \quad \boldsymbol{V}_{i}^{k}=\boldsymbol{K}_{i}^{k} \boldsymbol{W}_{i}^{k}, \quad i=1,2 .
$$

The matrices $\boldsymbol{K}_{i}^{k}$ are deduced from a Singular Value Decomposition of $\boldsymbol{L}_{i}^{k} \boldsymbol{G}_{i}^{k}$. Inserting (25) in the jump condition of (19) gives

$$
\boldsymbol{C}_{1}^{k} \boldsymbol{G}_{1}^{k} \boldsymbol{V}_{1}^{k}=\boldsymbol{C}_{2}^{k} \boldsymbol{G}_{2}^{k} \boldsymbol{V}_{2}^{k}
$$

Inserting (26) in this last equation gives

$$
\boldsymbol{C}_{1}^{k} \boldsymbol{G}_{1}^{k} \boldsymbol{K}_{1}^{k} \boldsymbol{W}_{1}^{k}=\boldsymbol{C}_{2}^{k} \boldsymbol{G}_{2}^{k} \boldsymbol{K}_{2}^{k} \boldsymbol{W}_{2}^{k}
$$

Setting

$$
\boldsymbol{S}_{i}^{k}=\boldsymbol{C}_{i}^{k} \boldsymbol{G}_{i}^{k} \boldsymbol{K}_{i}^{k} \quad i=1,2
$$

leads to

$$
\boldsymbol{S}_{1}^{k} \boldsymbol{W}_{1}^{k}=\boldsymbol{S}_{2}^{k} \boldsymbol{W}_{2}^{k}
$$

Then, the SVD resolution of the underdetermined system (30) gives

$$
\boldsymbol{W}_{2}^{k}=\left(\left(\boldsymbol{S}_{2}^{k}\right)^{-1} \boldsymbol{S}_{1}^{k} \mid \boldsymbol{R}_{S_{2}}^{k}\right)\left(\begin{array}{c}
\boldsymbol{W}_{1}^{k} \\
\boldsymbol{\Lambda}^{k}
\end{array}\right),
$$

where $\boldsymbol{R}_{S_{2}}^{k}$ is the kernel of $\boldsymbol{S}_{2}^{k}$, and $\boldsymbol{\Lambda}_{k}$ is a set of reals. Note that the same procedure can be applied to express $\boldsymbol{W}_{1}^{k}$ in terms of $\boldsymbol{W}_{2}^{k}$.

\subsection{General overview of the ESIM in 2D}

The basic idea of the ESIM can be summed up in three steps. Consider a time step $t_{n}$. Then

(1) on both sides of the interface $\Gamma$, we build smooth extensions $\boldsymbol{U}^{*}\left(x, y, t_{n}\right)$ of the exact solution $\boldsymbol{U}\left(x, y, t_{n}\right)$; 
(2) we estimate numerical values of $\boldsymbol{U}^{*}$ at the irregular points $\left(x_{i}, y_{j}\right)$, denoted by $\boldsymbol{U}_{i, j}^{*}$ and called modified values;

(3) we inject the $\boldsymbol{U}_{i, j}^{*}$ for time-marching at the irregular points on the other side of the interface.

More precisely, consider an irregular point $M\left(x_{I}, y_{J}\right)$ in $\Omega_{2}$, and its orthogonal projection $P$ on $\Gamma$ (figure 3 ). The coefficients of the two-dimensional $k$-th order Taylor expansions around $P$ are denoted by

$$
\boldsymbol{\Pi}_{i, j}^{k}=\left(1,\left(x_{i}-x_{P}\right),\left(y_{j}-y_{P}\right), \ldots, \frac{\left(y_{j}-y_{P}\right)^{k}}{k !}\right) .
$$

Then, from the notations (8) and (32), and for a given integer $k$, the modified value at $M\left(x_{I}, y_{J}\right)$ is

$$
\boldsymbol{U}^{*}\left(x_{I}, y_{J}, t_{n}\right)=\Pi_{I, J}^{k} \boldsymbol{U}_{1}^{k}
$$

Now, the key point is how to estimate $\boldsymbol{U}_{1}^{k}$ in (33). This is the goal of the next section. Note that, in accordance with (25) and (26), it amounts to estimate $\boldsymbol{W}_{1}^{k}$.

3.5 Numerical estimation of the solution and of its spatial derivatives at the interface

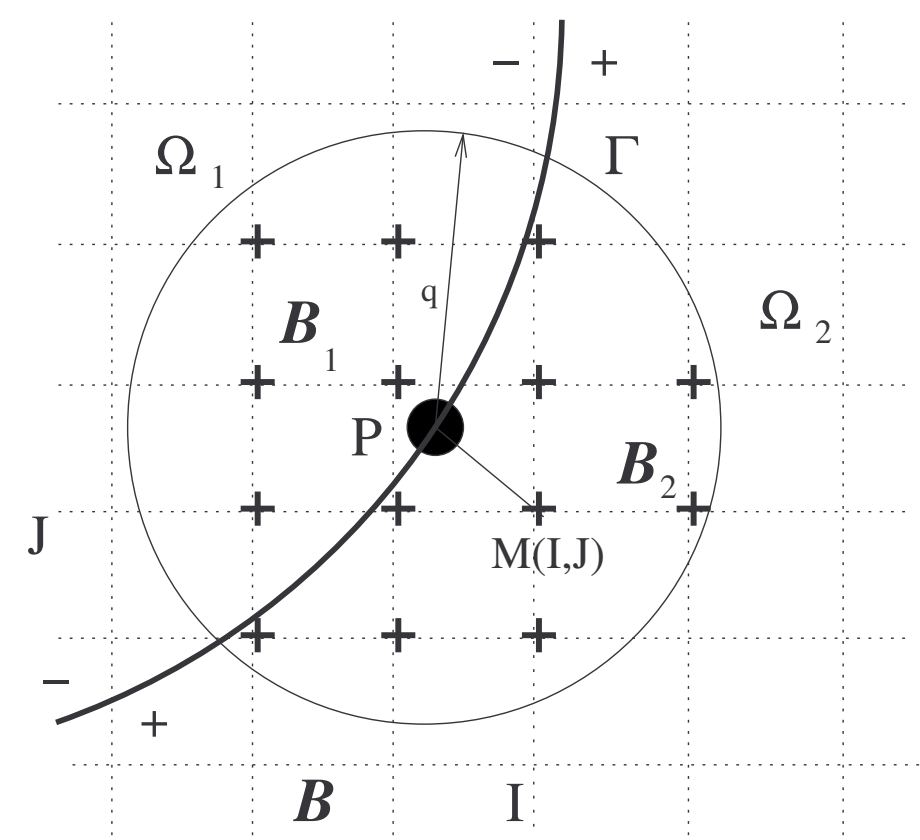

Fig. 3. Irregular point $M\left(x_{I}, y_{J}\right)$, orthogonal projection $P$ of $M$ on $\Gamma$, and the set of grid points $\mathcal{B}(+)$.

To estimate $\boldsymbol{W}_{1}^{k}$, we consider a set of grid points surrounding $P$. This set $\mathcal{B}$ is enclosed in the circle centered on $P$ with a radius $q$ ( $q$ is discussed further). 
$\mathcal{B}$ is divided into two subsets $\mathcal{B}_{i}$, according to the medium $\Omega_{i}(i=1,2)$.

Consider $\boldsymbol{U}\left(x_{i}, y_{j}, t_{n}\right)$ at the points of $\mathcal{B}$. We write their $k$-th order Taylor expansions at $P^{ \pm}$. If $(i, j)$ belongs to $\mathcal{B}_{1}$, we deduce from (25) and (26)

$$
\begin{aligned}
(i, j) \in \mathcal{B}_{1}, \boldsymbol{U}\left(x_{i}, y_{j}, t_{n}\right) & =\boldsymbol{\Pi}_{i, j}^{k} \boldsymbol{U}_{1}^{k}+\boldsymbol{O}\left(\Delta x^{k+1}\right) \\
& =\boldsymbol{\Pi}_{i, j}^{k} \boldsymbol{G}_{1}^{k} \boldsymbol{V}_{1}^{k}+\boldsymbol{O}\left(\Delta x^{k+1}\right) \\
& =\boldsymbol{\Pi}_{i, j}^{k} \boldsymbol{G}_{1}^{k} \boldsymbol{K}_{1}^{k} \boldsymbol{W}_{1}^{k}+\boldsymbol{O}\left(\Delta x^{k+1}\right) .
\end{aligned}
$$

Denoting by $\mathbf{1}$ and $\mathbf{0}$ respectively the identity matrix and the null matrix (whose dimensions depend of the configuration and are not detailed here), we deduce that

$$
(i, j) \in \mathcal{B}_{1}, \boldsymbol{U}\left(x_{i}, y_{j}, t_{n}\right)=\boldsymbol{\Pi}_{i, j}^{k} \boldsymbol{G}_{1}^{k} \boldsymbol{K}_{1}^{k}(\mathbf{1} \mid \mathbf{0})\left(\begin{array}{c}
\boldsymbol{W}_{1}^{k} \\
\\
\boldsymbol{\Lambda}^{k}
\end{array}\right)+\boldsymbol{O}\left(\Delta x^{k+1}\right)
$$

If $(i, j)$ belongs to $\mathcal{B}_{2}$, we deduce from $(25)$ and (26) that

$$
\begin{aligned}
(i, j) \in \mathcal{B}_{2}, \boldsymbol{U}\left(x_{i}, y_{j}, t_{n}\right) & =\boldsymbol{\Pi}_{i, j}^{k} \boldsymbol{U}_{2}^{k}+\boldsymbol{O}\left(\Delta x^{k+1}\right) \\
& =\boldsymbol{\Pi}_{i, j}^{k} \boldsymbol{G}_{2}^{k} \boldsymbol{V}_{2}^{k}+\boldsymbol{O}\left(\Delta x^{k+1}\right) \\
& =\boldsymbol{\Pi}_{i, j}^{k} \boldsymbol{G}_{2}^{k} \boldsymbol{K}_{2}^{k} \boldsymbol{W}_{2}^{k}+\boldsymbol{O}\left(\Delta x^{k+1}\right) .
\end{aligned}
$$

From this last equation and from (31), we deduce that

$$
\begin{gathered}
(i, j) \in \mathcal{B}_{2}, \boldsymbol{U}\left(x_{i}, y_{j}, t_{n}\right)=\boldsymbol{\Pi}_{i, j}^{k} \boldsymbol{G}_{2}^{k} \boldsymbol{K}_{2}^{k}\left(\left(\boldsymbol{S}_{2}^{k}\right)^{-1} \boldsymbol{S}_{1}^{k} \mid \boldsymbol{R}_{S_{2}}^{k}\right)\left(\begin{array}{c}
\boldsymbol{W}_{1}^{k} \\
\\
\boldsymbol{\Lambda}^{k}
\end{array}\right) \\
+\boldsymbol{O}\left(\Delta x^{k+1}\right) .
\end{gathered}
$$


The relations (35) and (37) are summed up via a matrix $\boldsymbol{M}$

$$
\left(\mathcal{U}^{n}\right)_{\mathcal{B}}=\boldsymbol{M}\left(\begin{array}{c}
\boldsymbol{W}_{1}^{k} \\
\\
\boldsymbol{\Lambda}^{k}
\end{array}\right)+\left(\begin{array}{c}
\boldsymbol{O}\left(\Delta x^{k+1}\right) \\
\vdots \\
\boldsymbol{O}\left(\Delta x^{k+1}\right)
\end{array}\right)
$$

where $\left(\mathcal{U}^{n}\right)_{\mathcal{B}}$ refers to the set of exact values $\boldsymbol{U}\left(x_{i}, y_{j}, t_{n}\right)$ at the points $(i, j)$ of $\mathcal{B}$. The radius $q$ of $\mathcal{B}$ is chosen so that (38) is overdetermined.

Then, we compute the least-squares inverse $\boldsymbol{M}^{-1}$ of $\boldsymbol{M}$ by classical techniques (normal equations, SVD, ...). For the sake of clarity, we will now confuse the exact values $\boldsymbol{W}_{1}^{k}$ and $\left(\mathcal{U}^{n}\right)_{\mathcal{B}}$ with their numerical estimations. We eliminate the Taylor rests in (38). Then, we get

$$
\left(\begin{array}{c}
\boldsymbol{W}_{1}^{k} \\
\boldsymbol{\Lambda}^{k}
\end{array}\right)=\boldsymbol{M}^{-1}\left(\mathcal{U}^{n}\right)_{\mathcal{B}}
$$

Since only $\boldsymbol{W}_{1}^{k}$ is of interest for us, a convenient restriction $\overline{\boldsymbol{M}^{-1}}$ of $\boldsymbol{M}^{-1}$ leads to

$$
\boldsymbol{W}_{1}^{k}=\overline{\boldsymbol{M}^{-1}}\left(\mathcal{U}^{n}\right)_{\mathcal{B}}
$$

\subsection{Computation of the modified values}

From (25), (26), and (33), we can write $\boldsymbol{U}_{I, J}^{*}$

$$
\begin{aligned}
(I, J) \in \Omega_{2}, \quad \boldsymbol{U}_{I, J}^{*} & =\boldsymbol{\Pi}_{I, J}^{k} \boldsymbol{U}_{1}^{k} \\
& =\boldsymbol{\Pi}_{I, J}^{k} \boldsymbol{G}_{1}^{k} \boldsymbol{K}_{1}^{k} \boldsymbol{W}_{1}^{k} .
\end{aligned}
$$

Then the modified value is deduced from (40)

$$
\boldsymbol{U}_{I, J}^{*}=\Pi_{I, J}^{k} \boldsymbol{G}_{1}^{k} \boldsymbol{K}_{1}^{k} \overline{\boldsymbol{M}^{-1}}\left(\mathcal{U}^{n}\right)_{\mathcal{B}}
$$

The same procedure is applied at each irregular point along $\Gamma$. For an irregular point on the $\Omega_{1}$ side, the matrices $\boldsymbol{G}_{1}^{k}$ and $\boldsymbol{K}_{1}^{k}$ are replaced by $\boldsymbol{G}_{2}^{k}$ and $\boldsymbol{K}_{2}^{k}$ in (42). Lastly, note that if $\Omega_{1}$ and $\Omega_{2}$ are respectively a fluid and a solid, the computation (42) involves both numerical values of the solution on the fluid side (with three components) and on the solid side (with five components). 
Then, the modified value $\boldsymbol{U}_{I, J}^{*}$ has three components (as an extension of the fluid solution), whereas $\boldsymbol{U}_{I, J}^{n}$ has five components (like the solution on the solid side).

\subsection{Time-stepping near interfaces}

Once all modified values have been computed at $t_{n}$, we can investigate the time-stepping at the irregular points. We use the same discrete operator $\boldsymbol{H}$ as at the regular points (12), but we modify some values that it uses. Consider an irregular point $(i, j)$ on one side of $\Gamma$. The discrete operator $\boldsymbol{H}$ now uses modified values at the grid points on the other side of $\Gamma$ than $(i, j)$, and numerical values at the grid points on the same side of $\Gamma$ than $(i, j)$.

To detail this time-stepping, we denote by $\Omega(i, j)$ the medium to which the point $(i, j)$ belongs. Then, we define the quantities $\tilde{\boldsymbol{U}}$ at the points of the stencil by

$$
\begin{aligned}
& \Omega(i+\alpha, j+\beta)=\Omega(i, j) \quad \Rightarrow \quad \tilde{\boldsymbol{U}}_{i+\alpha, j+\beta}=\boldsymbol{U}_{i+\alpha, j+\beta}^{n}, \\
& \Omega(i+\alpha, j+\beta) \neq \Omega(i, j) \quad \Rightarrow \quad \tilde{\boldsymbol{U}}_{i+\alpha, j+\beta}=\boldsymbol{U}_{i+\alpha, j+\beta}^{*} .
\end{aligned}
$$

Then, instead of (12), the time-stepping at an irregular point $(i, j)$ is now

$$
\boldsymbol{U}_{i, j}^{n+1}=\boldsymbol{H}\left(\tilde{\boldsymbol{U}}_{i+\alpha, j+\beta}\right) .
$$

Doing so is what we call the "Explicit Simplified Interface Method" (ESIM). Coupling the ESIM with a wide class of scheme is automatic: no modification of the scheme is required, and the computation of $\boldsymbol{U}_{i, j}^{*}$ 's does not depend on the discrete operator $\boldsymbol{H}$.

The ESIM incorporates into the scheme some insight about the geometry of $\Gamma$. Indeed, the derivation of the interface conditions (19) involves $x^{\prime}, y^{\prime}$ and their successive derivatives up to the $k$-th order at $P$. Moreover, the Taylor expansions (35) and (37) introduce a subcell resolution concerning the position of $P$ inside the meshing.

\subsection{Some implementation details}

Overview of the algorithm. For the sake of clarity, we sum up the interface method (in the following, $i=1,2, j=2$ if $i=1$, and $j=1$ if $i=2$ ). The algorithm can be divided into three parts: 
(1) Part 1: pre-processing step

- compute $\boldsymbol{G}_{i}^{k}(25), \boldsymbol{K}_{i}^{k}(26)$;

- find and store each irregular points along $\Gamma$;

- at each irregular point $M(I, J)$,

- find its orthogonal projection $P$ on $\Gamma$, find and store the points of $\mathcal{B}_{i}$;

- compute $\boldsymbol{C}_{i}^{k}, \boldsymbol{L}_{i}^{k}(19),\left(\left(\boldsymbol{S}_{j}^{k}\right)^{-1} \boldsymbol{S}_{i}^{k} \mid \boldsymbol{R}_{S_{j}}^{k}\right)(31)$

- fill $\boldsymbol{M}$ (38) from (35), (37), compute $\overline{\boldsymbol{M}^{-1}}$ (39);

- compute and store $\Pi_{I, J}^{k} \boldsymbol{G}_{j}^{k} \boldsymbol{K}_{j}^{k} \overline{\boldsymbol{M}^{-1}}$;

(2) Part 2: before each time step

- read each irregular point $M(I, J)$;

- at each irregular point $(I, J)$,

$-\operatorname{read} \mathcal{B}$ and $\boldsymbol{\Pi}_{I, J}^{k} \boldsymbol{G}_{j}^{k} \boldsymbol{K}_{j}^{k} \overline{\boldsymbol{M}^{-1}}$

- compute $\boldsymbol{U}_{I, J}^{*}(42)$;

(3) Part 3: at each time step

- at each point $(i, j)$, apply $(12)$ if $(i, j)$ is regular, or $(44)$ if $(i, j)$ is irregular.

Choice of the radius $q$. As mentioned in section 3.5, the radius $q$ of $\mathcal{B}$ is chosen such that (38) is overdetermined. Let $n_{d}$ be the number of available data, i.e. the number of components of $\left(\mathcal{U}^{n}\right)_{\mathcal{B}}$. Let too $n_{u}$ be the number of unknowns, i.e. the number of components of ${ }^{T}\left(\boldsymbol{W}_{1}^{k}, \boldsymbol{\Lambda}^{k}\right)$. Then, one must have

$$
n_{d}=K n_{u}, \quad K>1 .
$$

Note that $n_{d}$ depends on $q$ and on the local geometry of $\Gamma$ around $P$. Practically, $K=1.2$ seems to be the minimal value to use. To ensure (45) for each projection point $P$, two strategies are possible:

- use a constant $K$ and adapt $q$ to ensure (45), which may be cumbersome;

- use a constant $q$, and let vary $K$ Then, $q$ must ensure that $K \geq 1.2$ for all geometrical configurations around $P$. In some cases, one can obtain a number of data $n_{d}$ twice bigger as the required minimal value. 
For the sake of simplicity, we have chosen the second strategy, with

$$
q=3.5 \Delta x
$$

when $k=2$ (see the section 3.10$)$.

\subsection{Computational cost of the ESIM}

As an example, consider a numerical experiment with a plane vertical interface between two fluids. The computation is performed on a Personal Computer (Pentium III $800 \mathrm{MHz}$ ), with the Wave Propagation Algorithm (WPALG) coupled to the ESIM. The table 1 shows the number of irregular points for various values of $N_{x}$ and $N_{y}$ (the grid points in the $x$ and $y$ directions). It shows too the CPU time for the pre-processing step and for 100 time steps. Logically, the CPU time for Part 1 and for 100 Parts 2 grows linearly with $N_{x}$, whereas the CPU time for Part 3 grows quadratically. For $100 \times 100$ grid points, the CPU time for Part 2 amounts to $4 \%$ of the CPU time for Part 3; this ratio falls to $1 \%$ for $400 \times 400$ grid points. In conclusion, the computational cost induced by the ESIM is negligible compared with the cost of the scheme itself.

\begin{tabular}{c|cccc}
\hline$N_{x} \times N_{y}$ & Irregular points & Part 1 & Part 2 & Part 3 \\
\hline $100 \times 100$ & 384 & $3.7 \mathrm{~s}$ & $0.37 \mathrm{~s}$ & $9 \mathrm{~s}$ \\
& & & & \\
$200 \times 200$ & 784 & $7.4 \mathrm{~s}$ & $0.75 \mathrm{~s}$ & $36 \mathrm{~s}$ \\
$400 \times 400$ & 1584 & $15.3 \mathrm{~s}$ & $1.48 \mathrm{~s}$ & $144 \mathrm{~s}$ \\
\hline
\end{tabular}

Table 1

CPU times (in seconds) for the preprocessing step (Part 1) and for 100 computations of modified values (Part 2) and time-steppings (Part 3).

Note that the ESIM is a local treatment. It means two things about the computation of each modified value $\boldsymbol{U}_{I, J}^{*}$. First, it only involves the numerical values near the irregular point $(I, J)$. Second, it does not depend on the computation of the other modified values along $\Gamma$. As a consequence, the parallelization of the ESIM should be easy and efficient (providing that the scheme can be parallelized, of course). 


\subsection{Remarks about the numerical analysis}

We do not provide any theoretical result about the ESIM in 2D. The convergence analysis of a given scheme coupled with the ESIM is an interesting open question. However, we propose three remarks, deduced from many numerical experiments, and concerning successively the accuracy, the stability, and the case of non-smooth interfaces.

Accuracy. In 1D [22], we have analysed the local truncation error of the coupling between the ESIM and numerical schemes. In 2D and when the geometry of the interface is sufficiently smooth, we conjecture a similar result. We think that the coupling of a $r$-th order accurate scheme with the ESIM is still $r$-th order accurate at the irregular points if

$$
k \geq r
$$

As a consequence, we use $k=2$ for numerical experiments with second-order schemes such as Lax-Wendroff or WPALG (we recall that $k$ is the maximal order of the spatial derivations in the jump conditions).

Stability. The coupling of the ESIM with various schemes (Lax-Wendroff, Wave Propagation Algorithm, ...) has usually the same CFL limit of stability as the scheme in homogeneous medium, even for important contrasts of the physical parameters. As an example, we do not observe instabilities for the interface between water $\left(\rho_{0}=1000 \mathrm{~kg} / \mathrm{m}^{3}, c_{0}=1500 \mathrm{~m} / \mathrm{s}\right)$ and an elastic medium with higher density and sound speeds $\left(\rho_{1}=8500 \mathrm{~kg} / \mathrm{m}^{3}, c_{p 1}=6500\right.$ $\left.\mathrm{m} / \mathrm{s}, c_{s 1}=3250 \mathrm{~m} / \mathrm{s}\right)$. The previous physical parameters correspond to realistic values for existing solids (steel, aluminium, copper).

However, numerical instabilities occur for very high contrasts of the physical parameters such as the water-air interface (air: $\rho_{1}=1.3 \mathrm{~kg} / \mathrm{m}^{3}, c_{1}=340 \mathrm{~m} / \mathrm{s}$ ). In this case, the stability limit is around $\rho_{1}=100 \mathrm{~kg} / \mathrm{m}^{3}$ (with all the other parameters unchanged); below this limit, instabilities occur, with a minor influence of the CFL number and of $K(45)$.

Non-smooth interfaces. In subsection $2-1$, it is precised that only sufficiently smooth interfaces are considered. In view of the section 2 , it is clear that $\Gamma$ needs to be, at least, a $C^{k+1}$ curve. The case of less smooth interfaces is beyond the scope of the present paper.

However, our softwares can be adapted to non-sufficiently smooth interfaces, and then it is interesting to see what happens numerically. Sharp corners, where $x^{\prime}$ and $y^{\prime}$ are discontinuous, cannot be treated. On the contrary, the computations with $C^{1}$ and $C^{2}$ interfaces are apparently stable. The $C^{1}$ case corresponds e.g. to straight lines linked by arcs of circle; the $C^{2}$ case corre- 
sponds typically to cubic splines. Limitations concerning the contrasts of physical parameters, for stability purpose, are the same as for sufficiently smooth interfaces. The only restriction is that $x(\tau)$ and $y(\tau)$ must not vary too much on the scale of one mesh $\Delta x$.

Lastly and as an echo to the conjecture concerning the local truncation error, we let open the following question: what is the precision of one scheme coupled to the ESIM when the interface is not sufficiently smooth?

\section{Numerical experiments}

\subsection{The configurations}

To show the efficiency of the interface method, we focus on the fluid-solid case, because it presents many challenging difficulties. First, the number of interface conditions is smaller than the number of unknowns, which prevented the IIM from studying this case. Second, the number of unknowns differs on both sides of the interface $\Gamma$, which complicates the coupling between the two media. Moreover, the fluid-solid case offers a wide panel of interface conditions: two different compatibility conditions (21), (24), and boundary conditions only on the solid side. Numerical experiments with fluid-fluid or solid-solid interfaces can be found in [18].

In the examples, the fluid is $\Omega_{1}$, the solid is $\Omega_{2}$, and physical parameters are

$$
\begin{aligned}
& \rho_{1}=1000 \mathrm{~kg} / \mathrm{m}^{3}, \quad c_{1}=1500 \mathrm{~m} / \mathrm{s} \quad \text { if }(x, y) \in \Omega_{1}, \\
& \rho_{2}=2600 \mathrm{~kg} / \mathrm{m}^{3}, \quad c_{p 2}=4000 \mathrm{~m} / \mathrm{s}, \quad c_{s 2}=2000 \mathrm{~m} / \mathrm{s} \quad \text { if }(x, y) \in \Omega_{2} .
\end{aligned}
$$

The computations are initialized in the fluid $\Omega_{1}$ by a plane wave

$$
\boldsymbol{U}(x, y, t)=-{ }^{T}\left(\frac{\cos \theta_{1}}{c_{1}}, \frac{\sin \theta_{1}}{c_{1}}, \rho_{1}\right) f\left(t-\frac{x \cos \theta_{1}+y \sin \theta_{1}}{c_{1}}\right),
$$

where $\theta_{1}$ is the angle between the direction of propagation and the horizontal axis. The function $f$ is a $C^{2}$ spatially-bounded sinusoid

$$
f(\xi)= \begin{cases}\sin \left(\omega_{c} \xi\right)-\frac{1}{2} \sin \left(2 \omega_{c} \xi\right) & \text { if } 0<\xi<\frac{1}{f_{c}} \\ 0 \text { else. } & \end{cases}
$$


(a)

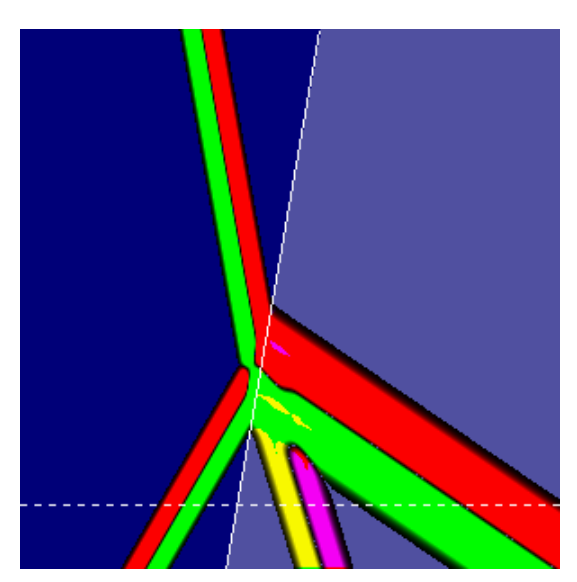

(c)

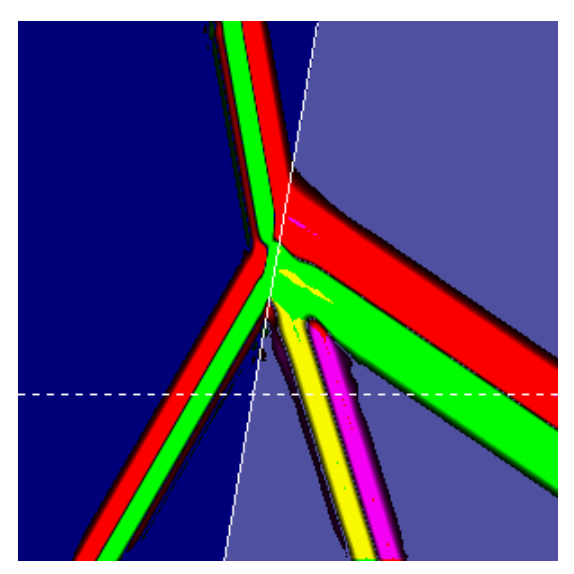

(e)

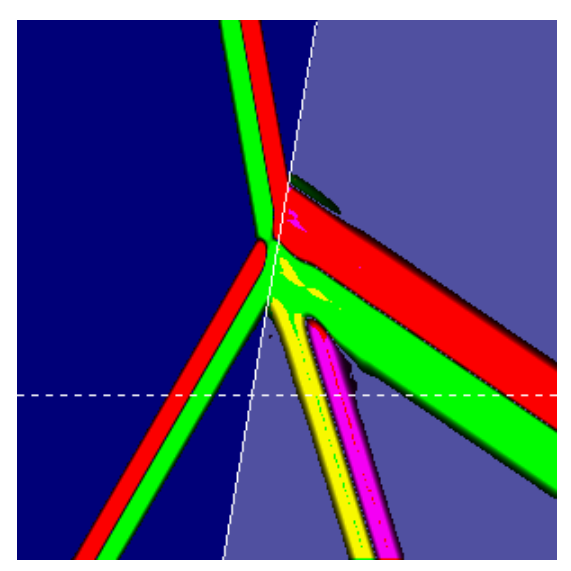

(b)

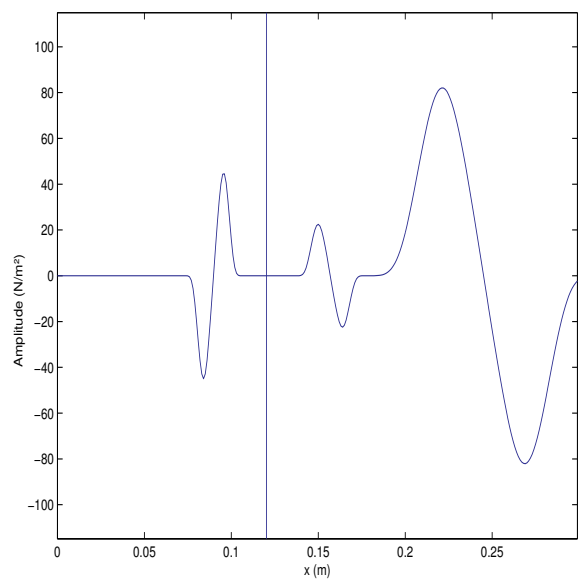

(d)

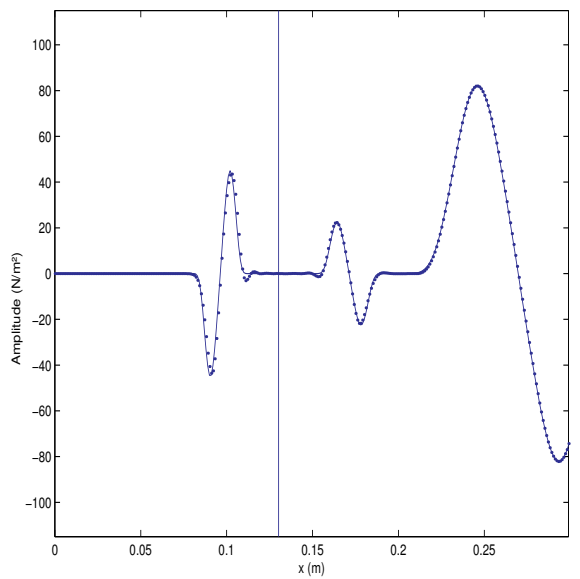

(f)

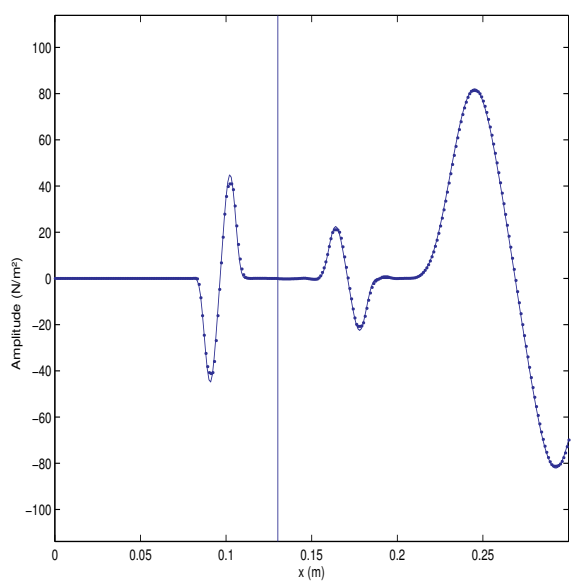

Fig. 4. Plane wave on a plane interface. Exact solution at initial instant (a-b), Lax-Wendroff + ESIM (c-d), WPALG + ESIM (e-f). Numerical solutions: points (d-f), exact solutions: solid line (b, d, f). 
We investigate three configurations: a plane interface, a circular interface, a circular shell. In each case, the numerical solutions are compared with analytical solutions. The analytical solution with a plane interface is not detailed, because it is straightforward [18]. The analytical solution with a circular interface is much more complicated and original; it is detailed in Appendix B. The analytical solution with a shell is not detailed, but one can deduce it easily from the approach shown in Appendix B.

In the circular case and the shell case, the exact solutions are in fact "semianalytical" solutions. There are indeed two approximations: the truncation of infinite Bessel series; the numerical approximations of inverse Fourier transforms. So, these exact solutions are used as a qualitative proof of convergence but not for convergence measures.

We use a green-red palette for P-waves (in fluids and solids) and a magentayellow palette for SV-waves (in solids). The distinction between these waves is based on a numerical estimation of div $\boldsymbol{v}$ and $\operatorname{curl} \boldsymbol{v}$. Note that the figures show $-p$ in the fluid and $\sigma_{11}$ in the solid.

\subsection{A plane interface}

Consider a $L_{x} \times L_{y}=0.3 \times 0.3 \mathrm{~m}^{2}$ domain. The interface $\Gamma$ is inclined $(\theta=$ 80 degrees with the horizontal axis), and the fluid $\Omega_{1}$ lies on the left of $\Gamma$. The incident $\mathrm{P}$-wave is defined by $\theta_{1}=21$ degrees (49), hence the incident plane wave crosses $\Gamma$ below the critical angle. Numerical experiments are performed with $N_{x} \times N_{y}=300 \times 300$ grid points and $f_{c}=\omega_{c} /(2 \pi)=510^{4} \mathrm{~Hz}$, hence 30 grid points by central wavelength in $\Omega_{1}$, and $\mathrm{CFL}=0.5$ in $\Omega_{2}$.

The figure 4 shows the exact solution at the initial instant $t_{0}=1.110^{-4} \mathrm{~s}(\mathrm{a}-\mathrm{b})$, and the numerical solution at $t_{1}=1.2510^{-4} \mathrm{~s}$ (after 125 time steps) with LaxWendroff (c-d) or WPALG (e-f), both coupled with the ESIM. The agreement between numerical values (points) and exact values (solid line) is excellent; the only differences are due to the scheme itself: numerical dispersion for LaxWendroff, numerical diffusion induced by limiters for WPALG. No spurious diffractions induced by the inclined interface $\Gamma$ are observed.

The table 2 shows measures of convergence obtained by refining the mesh. The schemes coupled with the ESIM are still second-order accurate, despite the non-smoothness of the solution across the interface $\Gamma$. 


$$
(\mathrm{i}=0)
$$

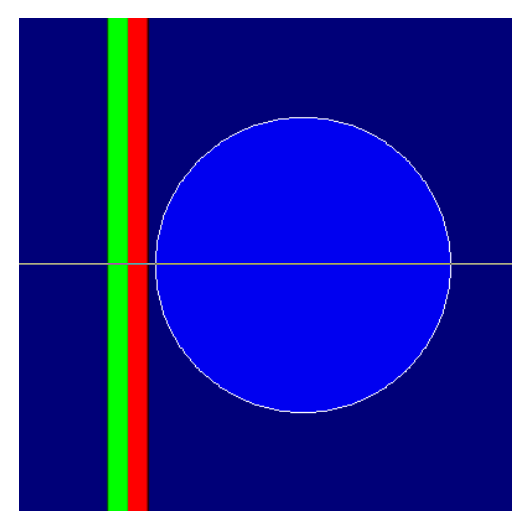

$$
(\mathrm{i}=1)
$$

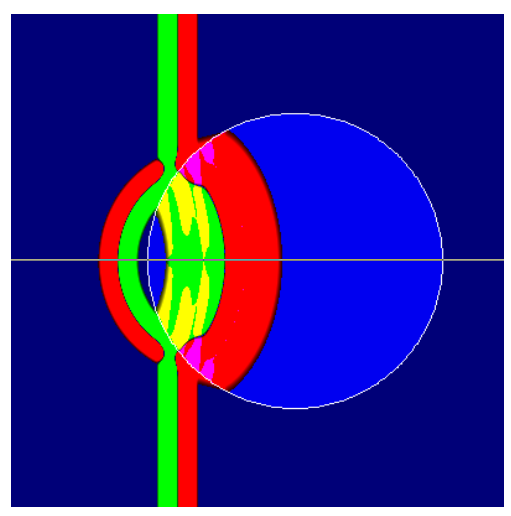

$$
(\mathrm{i}=2)
$$

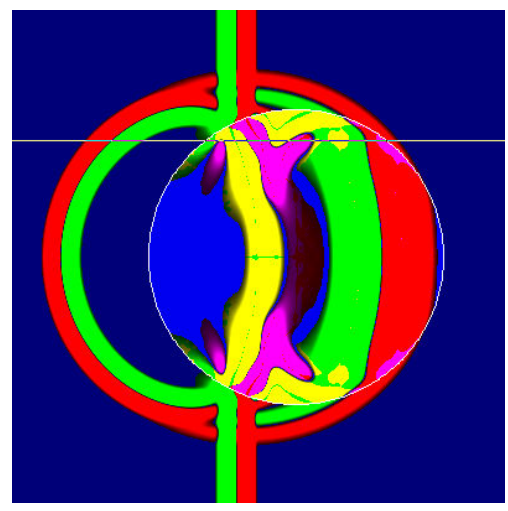

$(\mathrm{i}=0)$

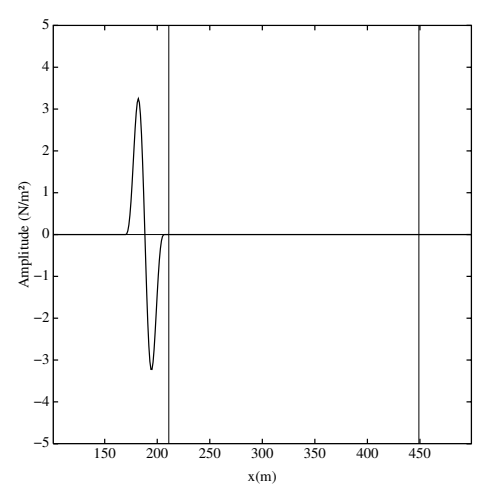

$(\mathrm{i}=1)$

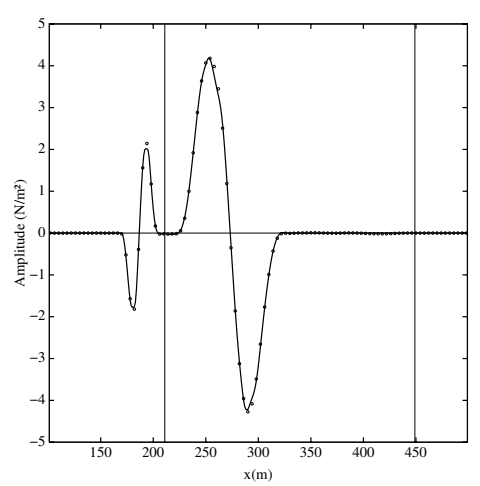

$(i=2)$

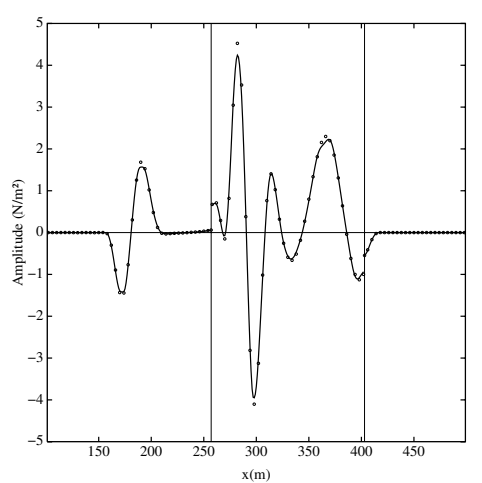

Fig. 5. Plane wave on a circular interface, with WPALG + ESIM (green-red: P-waves, magenta-yellow: SV-waves; exact solution: points, numerical solution: solid line). 

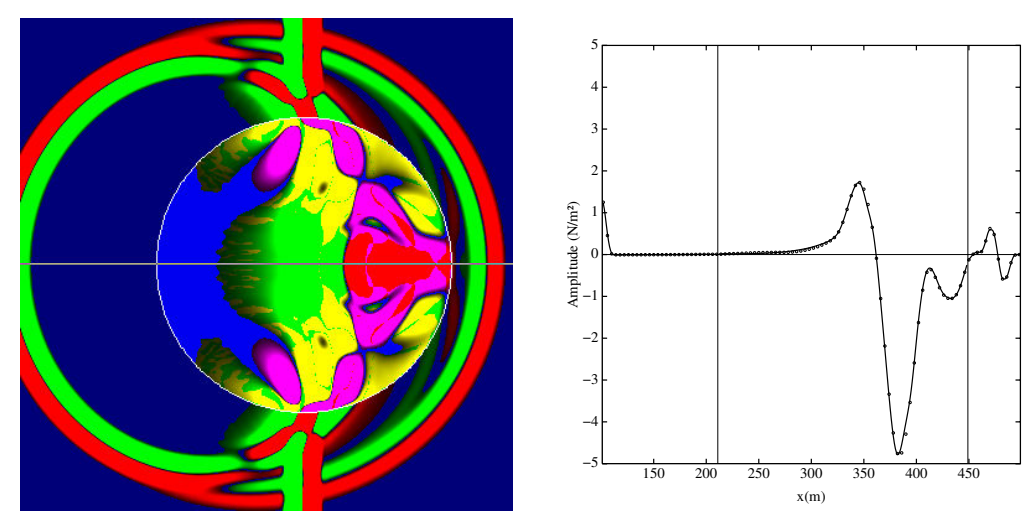

$(\mathrm{i}=4)$

$(\mathrm{i}=4)$
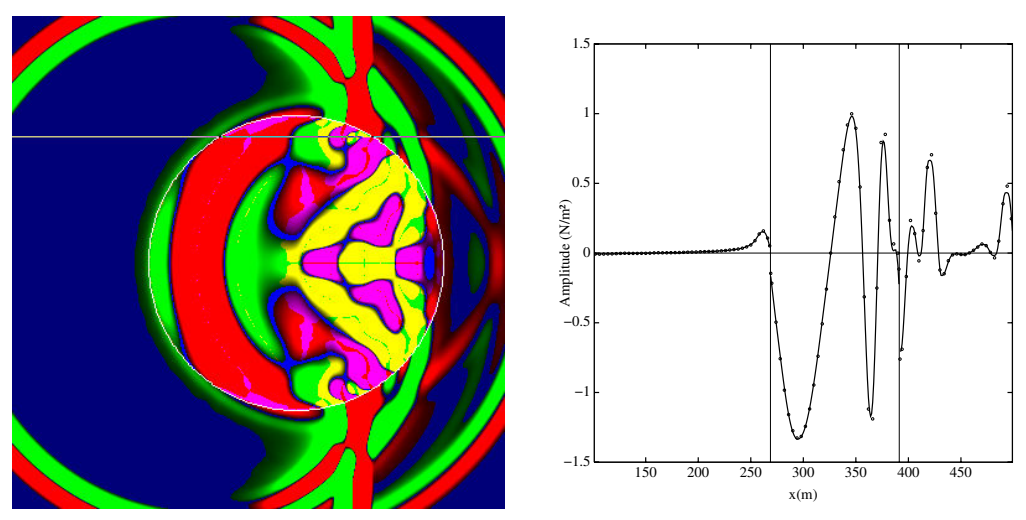

$(\mathrm{i}=5)$

$(\mathrm{i}=5)$
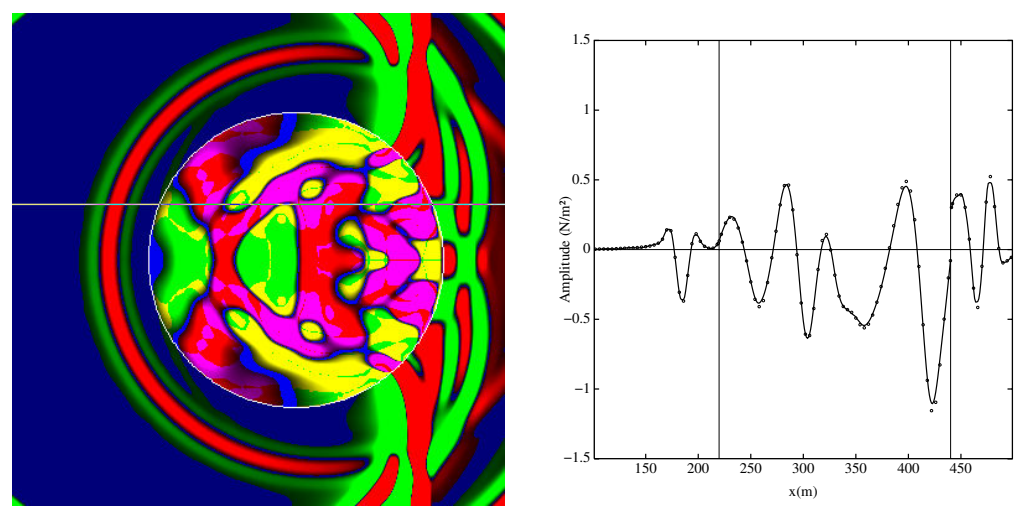

Fig. 6. Plane wave on a circular interface, with WPALG + ESIM ((green-red: P-waves, magenta-yellow: SV-waves; exact solution: points, numerical solution: solid line). 


\begin{tabular}{|c|c|c|c|c|c|}
\hline Scheme & $N_{x}$ & $\mathrm{~L}_{\infty}$ error & $\mathrm{L}_{\infty}$ order & $\mathrm{L}_{1}$ error & $\mathrm{L}_{1}$ order \\
\hline \multirow[t]{2}{*}{ Lax-Wendroff } & 100 & $2.51 \mathrm{e}+1$ & - & $8.33 \mathrm{e}-1$ & - \\
\hline & 200 & $8.73 \mathrm{e} 00$ & 1.52 & $2.48 \mathrm{e}-1$ & 1.74 \\
\hline \multirow[t]{2}{*}{+} & 400 & $2.61 \mathrm{e} 00$ & 1.74 & $6.01 \mathrm{e}-2$ & 2.04 \\
\hline & 800 & $6.31 \mathrm{e}-1$ & 2.04 & $1.50 \mathrm{e}-2$ & 2.00 \\
\hline \multirow[t]{2}{*}{ ESIM } & 1600 & $1.54 \mathrm{e}-1$ & 2.03 & $3.76 \mathrm{e}-3$ & 1.99 \\
\hline & 3200 & $3.89 \mathrm{e}-2$ & 1.98 & $9.46 \mathrm{e}-4$ & 1.97 \\
\hline \multirow[t]{2}{*}{ WPALG } & 100 & $1.05 \mathrm{e}+1$ & - & $3.15 \mathrm{e}-1$ & - \\
\hline & 200 & $4.08 \mathrm{e} 00$ & 1.36 & $8.56 \mathrm{e}-2$ & 1.88 \\
\hline \multirow[t]{2}{*}{+} & 400 & $1.33 \mathrm{e} 00$ & 1.61 & $2.02 \mathrm{e}-2$ & 2.08 \\
\hline & 800 & $4.42 \mathrm{e}-1$ & 1.59 & $5.30 \mathrm{e}-3$ & 1.93 \\
\hline \multirow[t]{2}{*}{ ESIM } & 1600 & $1.45 \mathrm{e}-1$ & 1.61 & $1.32 \mathrm{e}-3$ & 2.00 \\
\hline & 3200 & $4.83 \mathrm{e}-2$ & 1.58 & $3.41 \mathrm{e}-4$ & 1.95 \\
\hline
\end{tabular}

Table 2

Measures of convergence for a plane wave on a plane interface.

\subsection{A circular interface}

We consider a $L_{x} \times L_{y}=600 \times 600 \mathrm{~m}^{2}$ domain and a circular interface (radius $a=119 \mathrm{~m}$, centered at $\left.x_{0}=330 \mathrm{~m}, y_{0}=299 \mathrm{~m}\right)$. The fluid $\Omega_{1}$ is outside the circle. The incident $\mathrm{P}$-wave propagates horizontally $\left(\theta_{1}=0\right.$ degree (49)). The numerical experiments are performed with $N_{x} \times N_{y}=600 \times 600$ grid points and $\mathrm{CFL}=0.96$ in $\Omega_{2}$, with WPALG coupled with the ESIM. The central frequency is $f_{c}=40 \mathrm{~Hz}$.

The figures 5 and 6 show the numerical solutions at the initial instant $t_{0}=$ $0.138 \mathrm{~s}$ and at $t_{i}=t_{0}+130 i \Delta t(i=1, \ldots, 5)$, on a restricted domain $[100,500] \times$ $[100,500] \mathrm{m}^{2}$ centered on the middle of the computational domain. No special treatment is done to simulate the wave propagation in infinite medium (such 
as ABC's or PML's), but the times of integrations are sufficiently short to avoid that spurious waves reflected by the edges of the computational region are visible on the restricted domain (the same remark holds for the figures 1 , 7,8 , and 9). The horizontal line on each snapshot refers to the $y$-coordinate of the corresponding slice, where exact and numerical solutions are compared. These $y$-coordinates are respectively: $y=300 \mathrm{~m}(i=0), y=300 \mathrm{~m}(i=1)$, $y=393 \mathrm{~m}(i=2), y=300 \mathrm{~m}(i=3), y=401 \mathrm{~m}(i=4), y=344 \mathrm{~m}(i=5)$. The analytic solutions are computed with $N_{\text {Fourier }}=2^{16}$ and $N_{\text {Bessel }}=120$.

Classical wave phenomenons can be observed. The transmitted P-wave (greenred, $i=1$ ) is followed by the slower transmitted S-wave (yellow-magenta, $i=2$ ). The $\mathrm{SV}$-wave is minimal along the horizontal axis centered on $\Omega_{2}$ : this axis corresponds to a normal incidence of the incident plane wave, which is not converted in $\mathrm{P}$-wave. Headwaves (that is, waves that propagate faster in the fluid than the incident wave does [1]) are observed $(i=2,3)$. Then, refraction and conversion phenomenons are observed $(i=4,5)$.

The agreement between the numerical values and the exact values is excellent. The only differences are observed in the crests of the waves, where numerical values are slightly smaller than the exact ones (see e.g. figure $6, i=5$, near $x=425 \mathrm{~m}$ ). These differences are not caused by the interface treatment, but they are due to the numerical diffusion introduced by the flux-limiters of WPALG. To give evidence of this assertion, we note that the same drawback is observed when a wave has propagated in homogeneous medium (i.e. without any interface treatment) over a similar distance and with the same numerical parameters (such as the number of grid points for one wavelength). In the same order of idea, consider also the left part of the figure $4 \mathrm{f}$. At the instant of this snapshot, the reflected wave has not been subject to the influence of the interface treatment, and however one observes numerical diffusion (near $x=0.08 \mathrm{~m})$.

\subsection{A circular shell}

We consider an elastic shell immerged in water. Compared with the previous circular example, the only new parameter is the internal radius $a_{2}=89 \mathrm{~m}$. The main interest of this example is to discuss the stability of our method. As known, the multiple reflections inside the shell could lead to instable modes, see e.g. [7] in a slightly different context.

The numerical experiments are computed by WPALG coupled to the ESIM. The figures 7 and 8 show the numerical solutions at the initial instant $t_{0}=$ $0.138 \mathrm{~s}$ and $t_{i}=t_{0}+130 i \Delta t(i=1, \ldots, 5)$. The $y$-coordinates of the slices are respectively: $y=300 \mathrm{~m}(i=0), y=300 \mathrm{~m}(i=1), y=375 \mathrm{~m}(i=2)$, 
$(\mathrm{i}=0)$

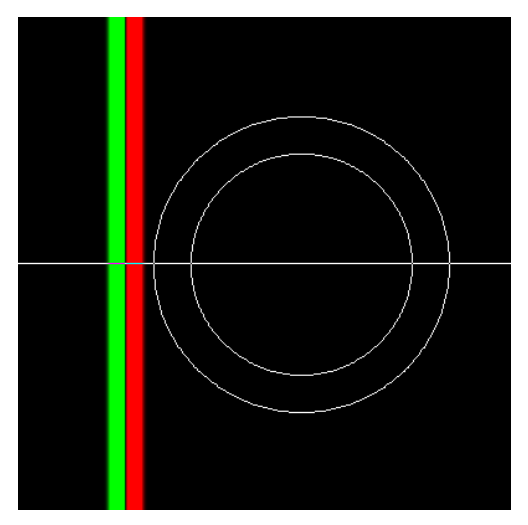

$(\mathrm{i}=1)$

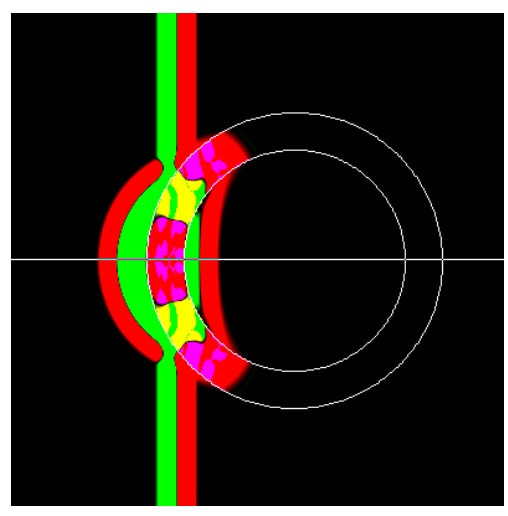

$(i=2)$

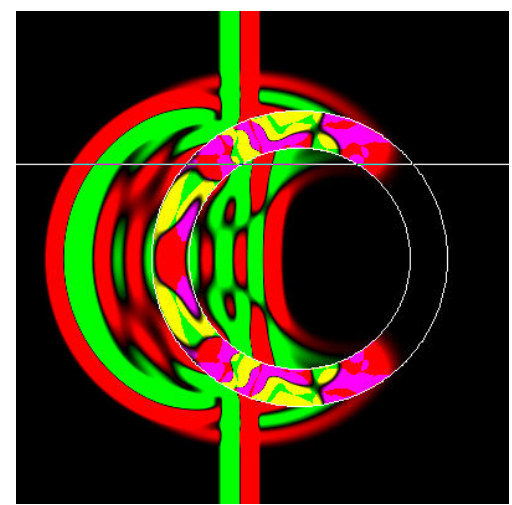

$(\mathrm{i}=0)$

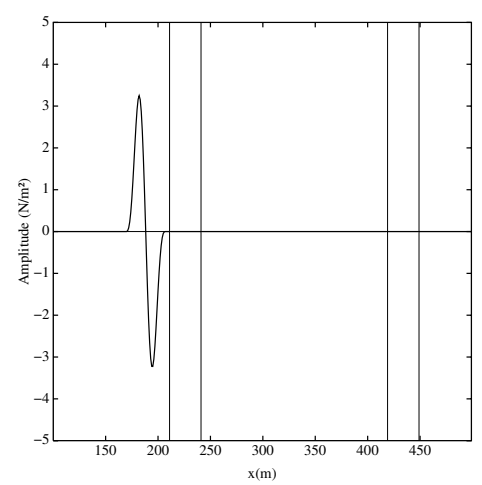

$(\mathrm{i}=1)$

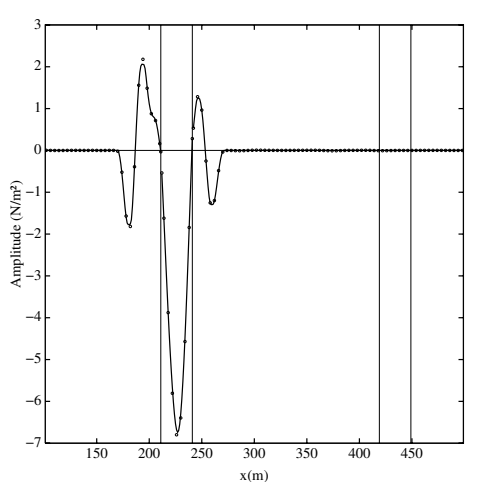

$(\mathrm{i}=2)$

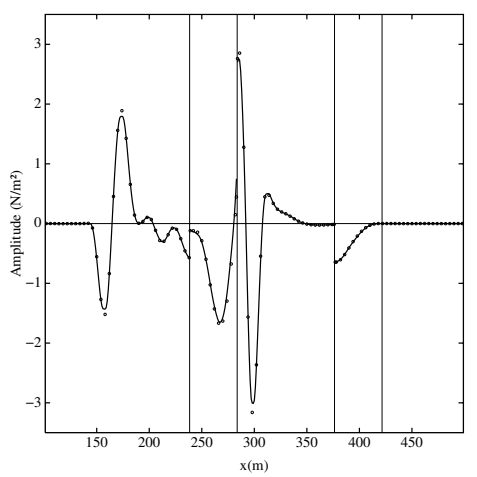

Fig. 7. Plane wave on a shell, with WPALG + ESIM (green-red: P-waves, magenta-yellow: SV-waves; exact solution: points, numerical solution: solid line). 

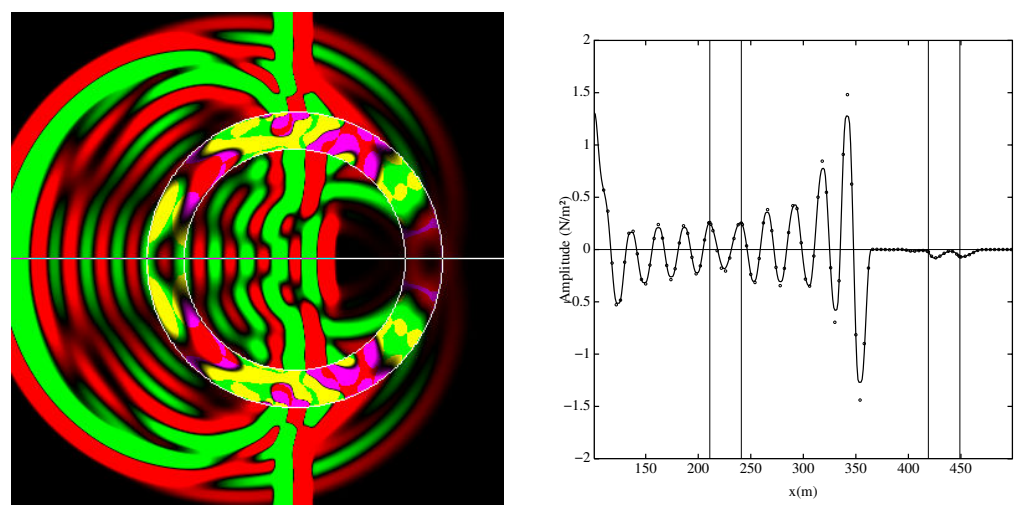

$$
(\mathrm{i}=4)
$$

$$
(\mathrm{i}=4)
$$
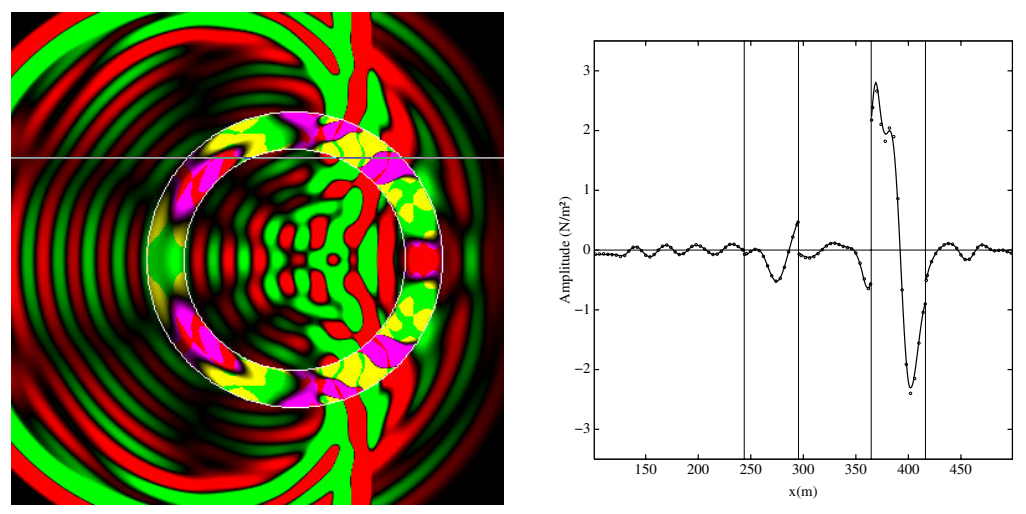

$$
(\mathrm{i}=5)
$$
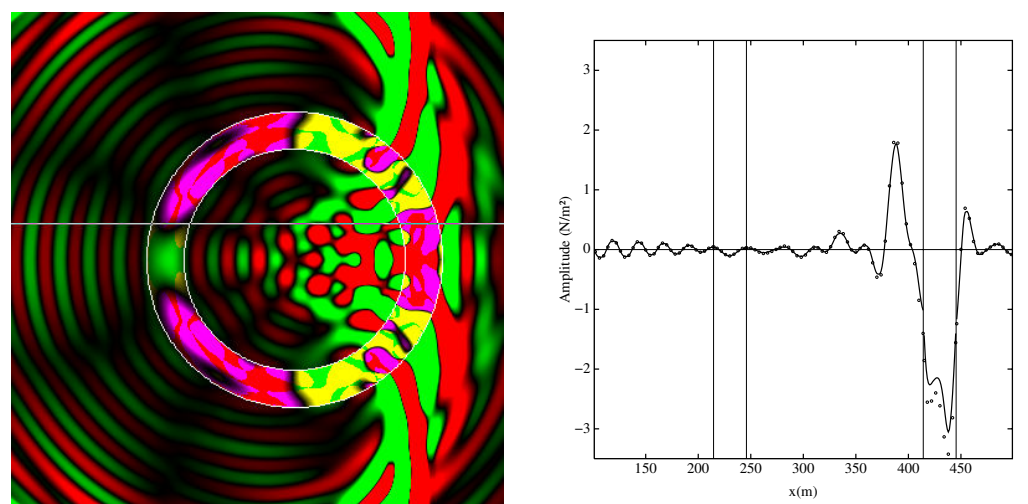

Fig. 8. Plane wave on a shell, with WPALG + ESIM (green-red: P-waves, magenta-yellow: SV-waves; exact solution: points, numerical solution: solid line). 
$y=300 \mathrm{~m}(i=3), y=381 \mathrm{~m}(i=4), y=328 \mathrm{~m}(i=5)$. The agreement between numerical and exact values is excellent. No instabilities are observed, even for these long time simulations.

(a)

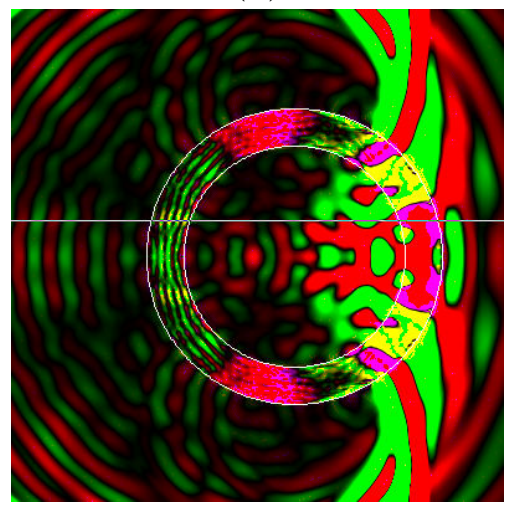

(b)

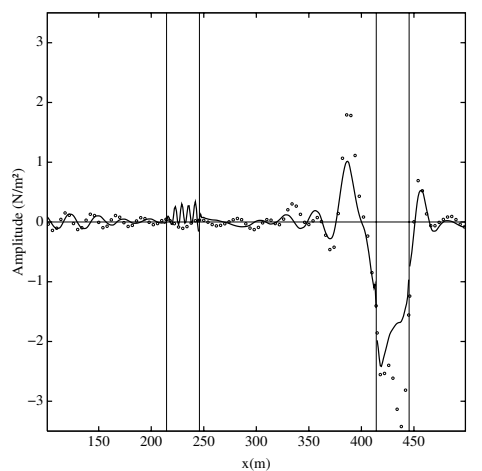

Fig. 9. Plane wave on a shell, without an interface treatment (green-red: P-waves, magenta-yellow: SV-waves; exact solution: points, numerical solution: solid line).

As a last example, we show in figure 9 what happens without an interface treatment, at the same instant than in figure $8(i=5)$. Spurious waves and instabilities are observed. The agreement between exact and numerical values is bad. For longer time of integration, instabilities destroy the computation.

\section{Conclusion}

We have presented a numerical method to treat two-dimensional interface problems in acoustics and elastodynamics. This method, called the "Explicit Simplified Interface Method" (ESIM), has three goals. First, the ESIM maintains properties of the schemes in homogeneous medium. Second, the ESIM takes into account complex geometries on a uniform Cartesian grid. Third, the ESIM incorporates in numerical schemes the jump conditions and the boundary conditions satisfied by the exact solution. The algorithm is easy to couple with a wide class of schemes, such as the Wave Propagation Algorithm (WPALG) [14], for a negligible computational cost. The coupling has been tested successfully on a challenging case: the fluid-solid interface, in three configurations.

This interface method is a priori not restricted to the two-dimensional configurations considered during this paper. Its extension to three-dimensional cases should be straightforward. The ESIM should be also of great interest for other linear interface problems, such as the advection or the Maxwell equations [4] with interfaces. 
The extension of the ESIM to nonlinear problems is a challenging project. We think to CFD problems, such as the Navier-Stokes equations [15] or the Euler equations with moving interfaces. We have especially in mind the numerical treatment of material interfaces in multicomponent flows. The "Ghost Fluid Method" of Fedkiw and al. [5] is efficient and robust in that context, but it is only first-order accurate. Moreover, it does not take into account precisely the geometrical features of the interface. The ESIM approach could bring noticeable improvements.

\section{A Details about the interface conditions}

First, we detail the matrices $\boldsymbol{G}_{i}^{k}$ deduced from (21), (24), and used in (25). If $\Omega_{i}$ is a fluid, the non-null components of $\boldsymbol{G}_{i}^{k}$ are

$$
\begin{aligned}
& \alpha=0, \quad \beta=0, \\
& \text { for } \gamma=0, . ., k \\
& \text { for } \varepsilon=1, \ldots, 3 \\
& \alpha=\alpha+1, \quad \beta=\beta+1, \quad G_{i}^{k}[\alpha, \beta]=1 \\
& \text { for } \delta=1, \ldots, \gamma \\
& \alpha=\alpha+1, \quad \beta=\beta-1, \quad \boldsymbol{G}_{i}^{k}[\alpha, \beta]=1 \\
& \alpha=\alpha+1, \quad \beta=\beta+2, \quad G_{i}^{k}[\alpha, \beta]=1 \\
& \alpha=\alpha+1, \quad \beta=\beta+1, \quad G_{i}^{k}[\alpha, \beta]=1 .
\end{aligned}
$$


If $\Omega_{i}$ is a solid, the non-null components of $G_{i}^{k}$ are

$$
\begin{aligned}
& \begin{array}{l}
\alpha=0, \quad \beta=0, \\
\text { for } \gamma=0, \ldots, k, \text { for } \delta=0, \ldots, \gamma
\end{array} \\
& \text { if } \delta=0 \text { then for } \varepsilon=1, \ldots, 5 \\
& \alpha=\alpha+1, \quad \beta=\beta+1, \quad \boldsymbol{G}_{i}^{k}[\alpha, \beta]=1 \\
& \text { if } \gamma \neq 0 \text { and } \delta \neq 0 \text { and } \gamma \neq \delta \text { then } \\
& \text { if } \gamma=2 \text { then } \nu=0, \eta=0 \text {, } \\
& \text { else if } \delta=1 \text { then } \nu=0, \eta=1 \text {, } \\
& \text { else if } \delta=\gamma-1 \text { then } \nu=1, \eta=0 \text {, } \\
& \text { else } \nu=1, \eta=1 \text {, } \\
& \alpha=\alpha+1, \quad \beta=\beta+1, \quad G_{l}^{k}[\alpha, \beta]=1 \\
& \alpha=\alpha+1, \quad \beta=\beta+1, \quad G_{l}^{k}[\alpha, \beta]=1 \\
& \alpha=\alpha+1, \quad \beta=\beta+1, \quad \boldsymbol{G}_{l}^{k}[\alpha, \beta]=1 \\
& \alpha=\alpha+1, \quad \beta=\beta-5+\nu, \quad \boldsymbol{G}_{l}^{k}[\alpha, \beta]=\alpha_{2} \\
& \beta=\beta+2-\nu, \quad \boldsymbol{G}_{l}^{k}[\alpha, \beta]=\alpha_{1} \\
& \beta=\beta+7, \quad G_{l}^{k}[\alpha, \beta]=\alpha_{1} \\
& \beta=\beta+2-\eta, \quad G_{l}^{k}[\alpha, \beta]=\alpha_{2} \\
& \alpha=\alpha+1, \quad \beta=\beta-5+\eta, \quad \boldsymbol{G}_{l}^{k}[\alpha, \beta]=1 \\
& \alpha=\alpha+1, \quad \beta=\beta+1, \quad G_{l}^{k}[\alpha, \beta]=1 .
\end{aligned}
$$

Second, we shortly recall how to solve an underdetermined system

$$
\boldsymbol{A} \boldsymbol{x}=\boldsymbol{y}
$$

by Singular Value Decomposition (SVD), where $\boldsymbol{A}$ is a $m \times n$ matrix $(m<n)$. $\boldsymbol{A}$ is splitted in [23]

$$
\boldsymbol{A}=\boldsymbol{X}_{A} \boldsymbol{Y}_{A}^{T} \boldsymbol{Z}_{A}
$$


where

- $\boldsymbol{X}_{A}$ is a $m \times n$ orthogonal matrix,

- $\boldsymbol{Y}_{A}$ is a $n \times n$ diagonal matrix of singular values with $n-m$ zeroes,

- $Z_{A}$ is a $n \times n$ orthonormal matrix.

This case is encountered in section 3.3. The least-squares inverse $\boldsymbol{A}^{-1}$ of $\boldsymbol{A}$ is

$$
\boldsymbol{A}^{-1}=\boldsymbol{Z}_{A} \boldsymbol{Y}_{A}^{-1 T} \boldsymbol{X}_{A}
$$

with $\boldsymbol{Y}_{A}^{-1}[i, i]=1 / \boldsymbol{Y}_{A}[i, i]$ if $\boldsymbol{Y}_{A}[i, i] \neq 0,0$ else $(i=1, \ldots, n)$. The full span of solutions of (A.3) is given by

$$
\boldsymbol{x}=\boldsymbol{A}^{-1} \boldsymbol{y}+\boldsymbol{x}_{0}
$$

where $\boldsymbol{x}_{0}$ is a linear combination of the column vectors $\boldsymbol{z}$ of $\boldsymbol{Z}_{A}$ whose samenumbered singular values are equal to zero. Suppose that the null singular values are numbered from $n-m+1$ to $n$. Then,

$$
\begin{aligned}
\boldsymbol{x}_{0} & =\lambda_{n-m+1} \boldsymbol{z}_{n-m+1}+\ldots+\lambda_{n} \boldsymbol{z}_{n} \\
& =\boldsymbol{R}_{A} \boldsymbol{\Lambda},
\end{aligned}
$$

where $\boldsymbol{R}_{A}$ is the restriction of $\boldsymbol{Z}_{A}$ filled with the vectors $\boldsymbol{z}_{n-m+1}, \ldots, \boldsymbol{z}_{n}$, and $\Lambda={ }^{T}\left(\lambda_{n-m+1}, \ldots, \lambda_{n}\right) \in \mathbb{R}^{n-m}$.

\section{B Exact solution for a plane wave on a fluid-solid circular interface}

This analytical solution is obtained in 6 steps:

(1) Fourier transform of the incident wave (50);

(2) writing the acoustic and elastic potentials on a basis of circular functions;

(3) expression of the acoustic and elastic fields from their potentials;

(4) computation of the reflection and transmission coefficients from (10);

(5) come-back in cartesian coordinates;

(6) inverse Fourier transform of the acoustic and elastic fields.

Step 1. The notations are the same than in section 4.3. The circular interface with a radius $a$ is centered at $\left(x_{0}, y_{0}\right)$. The angular frequency is $\omega$, the wavenumbers are

$$
k_{1}=\frac{\omega}{c_{1}}, \quad k_{p 2}=\frac{\omega}{c_{p 2}}, \quad k_{s 2}=\frac{\omega}{c_{s 2}} .
$$


The Fourier transform $A(\omega)$ of $f(50)$ is

$$
A(\omega)=\frac{\omega_{c}}{2 \pi}\left(\frac{1}{\omega^{2}-\omega_{c}^{2}}+\frac{1}{\omega^{2}-4 \omega_{c}^{2}}\right)\left(e^{-i \frac{2 \pi}{\omega_{c}} \omega}-1\right) .
$$

Step 2. Consider a point $M \in \Omega_{1}$, with coordinates $M\left(x=x_{0}+r \cos \phi, y=\right.$ $\left.y_{0}+r \sin \phi\right)$. The potential of the acoustic harmonic incident plane wave at $M$ is

$$
\begin{aligned}
\Phi_{i n c}(x, y, \omega) & =A e^{i\left(\omega t-k_{1}\left(x \cos \theta_{1}+y \sin \theta_{1}\right)\right)} \\
& =A e^{i \omega t} e^{-i k_{1}\left(x_{0} \cos \theta_{1}+y_{0} \sin \theta_{1}\right)} e^{-i k_{1} r \cos \left(\phi-\theta_{1}\right)} .
\end{aligned}
$$

For the sake of clarity, the time dependance $e^{i \omega t}$ is removed. We denote

$$
S=e^{-i k_{1}\left(x_{\Omega} \cos \theta_{1}+y_{\Omega} \sin \theta_{1}\right)}, \quad \theta=\phi-\theta_{1} .
$$

Then, the classical property of first-kind Bessel functions $J_{n}[20]$

$$
e^{i r \cos \theta}=\sum_{n=0}^{+\infty} \varepsilon_{n} i^{n} \cos n \theta J_{n}(r)
$$

(with $\varepsilon_{n}=1$ if $n=0,2$ else) leads to

$$
\Phi_{i n c}(x, y, \omega)=A S \sum_{n=0}^{+\infty} \varepsilon_{n} i^{n} \cos n \theta J_{n}\left(-k_{1} r\right) .
$$

To satisfy the Sommerfeld condition, the acoustic potential $\Phi_{\text {ref }}$ of the reflected wave is written on a basis of first-kind Hankel functions. Lastly and to avoid any singularity at $r=0$, the elastic potentials $\Phi_{t r a}^{p}$ of the transmitted P waves and the elastic pseudo-potential $\Psi_{t r a}^{s}=\left(0,0, \Psi_{t r a}^{s}\right)$ of the transmitted $\mathrm{SV}$ waves are written on a basis of first-kind Bessel functions. Hence, we write

$$
\begin{aligned}
\Phi_{r e f} & =\sum_{n=0}^{+\infty} R_{n} \cos n \theta H_{n}\left(-k_{1} r\right), \\
\Phi_{\text {tra }}^{p} & =\sum_{n=0}^{+\infty} T_{n}^{p} \cos n \theta J_{n}\left(-k_{p 2} r\right), \\
\Psi_{t r a}^{s} & =\sum_{n=0}^{+\infty} T_{n}^{s} \sin n \theta J_{n}\left(-k_{s 2} r\right),
\end{aligned}
$$

where $R_{n}, T_{n}^{p}$ and $T_{n}^{s}$ are the unknown coefficients of reflection and transmission, to be determined. 
Step 3. In a fluid, the acoustic velocity $\boldsymbol{v}={ }^{T}\left(v_{r}, v_{\theta}\right)$ and the acoustic pressure are deduced from the potential $\Phi$ by

$$
\boldsymbol{v}=\operatorname{grad} \Phi, \quad p=-\rho \frac{\partial \Phi}{\partial t} .
$$

In a solid, the elastic displacement $\boldsymbol{u}={ }^{T}\left(u_{r}, u_{\theta}\right)$ is deduced from $\Phi$ (for P-waves) or from $\boldsymbol{\Psi}=(0,0, \Psi)$ (for $\mathrm{SV}$-waves) by

$$
\boldsymbol{u}=\operatorname{grad} \Phi \text { for } \mathrm{P} \text {-waves, } \quad \boldsymbol{u}=\operatorname{curl} \Psi \text { for } \mathrm{SV} \text {-waves. }
$$

The elastic velocity $\boldsymbol{v}=\frac{\partial \boldsymbol{u}}{\partial t}$ and the three independant components of the stress tensor in cylindrical coordinates are deduced from $\boldsymbol{u}$ [20]

$$
\begin{aligned}
& \sigma_{r r}=(\lambda+2 \mu) \frac{\partial u_{r}}{\partial r}+\lambda\left(\frac{u_{r}}{r}+\frac{1}{r} \frac{\partial u_{\theta}}{\partial \theta}\right), \\
& \sigma_{r \theta}=\mu\left(\frac{\partial u_{\theta}}{\partial r}-\frac{u_{\theta}}{r}+\frac{1}{r} \frac{\partial u_{r}}{\partial \theta}\right), \\
& \sigma_{\theta \theta}=(\lambda+2 \mu)\left(\frac{1}{r} \frac{\partial u_{\theta}}{\partial \theta}+\frac{u_{r}}{r}\right)+\lambda \frac{\partial u_{r}}{\partial r} .
\end{aligned}
$$

In cylindrical coordinates, the grad and curl operators are

$$
\operatorname{grad} \Phi=\left(\begin{array}{c}
\frac{\partial \Phi}{\partial r} \\
\frac{1}{r} \frac{\partial \Phi}{\partial \theta}
\end{array}\right), \quad \operatorname{curl} \Psi(0,0, \Psi)=\left(\begin{array}{c}
\frac{1}{r} \frac{\partial \Psi}{\partial \theta} \\
-\frac{\partial \Psi}{\partial r}
\end{array}\right)
$$

From (B.6), (B.7), (B.8), and (B.10), we easily deduce the harmonic fields on the whole domain. The components of the incident $\mathrm{P}$ wave are

$$
\begin{aligned}
& v_{r}^{i n c}=\frac{1}{c_{1}} i \omega A S \sum_{n=0}^{+\infty} \varepsilon_{n} i^{n+1} \cos n \theta J_{n}^{\prime}\left(-k_{1} r\right), \\
& v_{\theta}^{i n c}=i \omega A S \sum_{n=0}^{+\infty} \varepsilon_{n} i^{n+1} \frac{n \sin n \theta}{r \omega} J_{n}\left(-k_{1} r\right), \\
& p^{i n c}=-\rho_{1} i \omega A S \sum_{n=0}^{+\infty} \varepsilon_{n} i^{n} \cos n \theta J_{n}\left(-k_{1} r\right) .
\end{aligned}
$$

The components of the reflected $\mathrm{P}$ wave are 


$$
\begin{aligned}
& v_{r}^{r e f}=\frac{1}{c_{1}} i \omega \sum_{n=0}^{+\infty} i R_{n} \cos n \theta H_{n}^{\prime}\left(-k_{1} r\right), \\
& v_{\theta}^{r e f}=i \omega \sum_{n=0}^{+\infty} i R_{n} \frac{n \sin n \theta}{r \omega} H_{n}\left(-k_{1} r\right), \\
& p^{r e f}=-\rho_{1} i \omega \sum_{n=0}^{+\infty} R_{n} \cos n \theta H_{n}\left(-k_{1} r\right) .
\end{aligned}
$$

The components of the transmitted $\mathrm{P}$ wave are

$$
\begin{aligned}
& v_{r}^{p}= \frac{1}{c_{p 2}} i \omega \sum_{n=0}^{+\infty} i T_{n}^{p} \cos n \theta J_{n}^{\prime}\left(-k_{p 2} r\right) \\
& v_{\theta}^{p}=i \omega \sum_{n=0}^{+\infty} i T_{n}^{p} \frac{n \sin n \theta}{r \omega} J_{n}\left(-k_{p 2} r\right) \\
& \sigma_{r r}^{p}=-i \omega \sum_{n=0}^{+\infty} T_{n}^{p} \frac{\cos n \theta}{\omega^{2}}\left((\lambda+2 \mu) k_{p 2}^{2} J_{n}^{\prime \prime}\left(-k_{p 2} r\right)\right. \\
&\left.\quad-\lambda \frac{k_{p 2}}{r} J_{n}^{\prime}\left(-k_{p 2} r\right)-\lambda\left(\frac{n}{r}\right)^{2} J_{n}\left(-k_{p 2} r\right)\right) \\
& \sigma_{r \theta}^{p}=-2 \mu i \omega \sum_{n=0}^{+\infty} T_{n}^{p} \frac{\sin n \theta}{\omega^{2}}\left(\frac{n}{r^{2}} J_{n}\left(-k_{p 2} r\right)+n \frac{k_{p 2}}{r} J_{n}^{\prime}\left(-k_{p 2} r\right)\right) \\
& \sigma_{\theta \theta}^{p}=-i \omega \sum_{n=0}^{+\infty} T_{n}^{p} \frac{\cos n \theta}{\omega^{2}}\left(\lambda k_{p 2}^{2} J_{n}^{\prime \prime}\left(-k_{p 2} r\right)-(\lambda+2 \mu) \frac{k_{p 2}}{r} J_{n}^{\prime}\left(-k_{p 2} r\right)\right. \\
&\left.\quad-(\lambda+2 \mu)\left(\frac{n}{r}\right)^{2} J_{n}\left(-k_{p 2} r\right)\right)
\end{aligned}
$$

Lastly, the components of the transmitted $\mathrm{S}$ wave are

$$
\begin{aligned}
v_{r}^{s} & =-i \omega \sum_{n=0}^{+\infty} i T_{n}^{s} \frac{n \cos n \theta}{r \omega} J_{n}\left(-k_{s 2} r\right), \\
v_{\theta}^{s} & =-\frac{1}{c_{s 2}} i \omega \sum_{n=0}^{+\infty} i T_{n}^{s} \sin n \theta J_{n}^{\prime}\left(-k_{s 2} r\right), \\
\sigma_{r r}^{s} & =2 \mu i \omega \sum_{n=0}^{+\infty} T_{n}^{s} \frac{\cos n \theta}{\omega^{2}}\left(\frac{n}{r^{2}} J_{n}\left(-k_{s 2} r\right)+n \frac{k_{s 2}}{r} J_{n}^{\prime}\left(-k_{s 2} r\right)\right), \\
\sigma_{r \theta}^{s} & =\mu i \omega \sum_{n=0}^{+\infty} T_{n}^{s} \frac{\sin n \theta}{\omega^{2}}\left(k_{s 2}^{2} J_{n}^{\prime \prime}\left(-k_{s 2} r\right)+\frac{k_{s 2}}{r} J_{n}^{\prime}\left(-k_{s 2} r\right)+\left(\frac{n}{r}\right)^{2} J_{n}\left(-k_{s 2} r\right)\right), \\
\sigma_{\theta \theta}^{s} & =-2 \mu i \omega \sum_{n=0}^{+\infty} T_{n}^{s} \frac{n \cos n \theta}{\omega^{2}}\left(\frac{k_{s 2}}{r} J_{n}^{\prime}\left(-k_{s 2} r\right)+\frac{1}{r^{2}} J_{n}\left(-k_{s 2} r\right)\right) .
\end{aligned}
$$


Step 4. We compute the coefficients $R_{n}, T_{n}^{p}$ and $T_{n}^{s}$. To do so, we deduce from the interface conditions (10) that

$$
\begin{aligned}
& \left(v_{r}^{i n c}+v_{r}^{r e f}\right)\left(a^{+}, \theta\right)=\left(v_{r}^{p}+v_{r}^{s}\right)\left(a^{-}, \theta\right), \\
& -\left(p^{i n c}+p^{r e f}\right)\left(a^{+}, \theta\right)=\left(\sigma_{r r}^{p}+\sigma_{r r}^{s}\right)\left(a^{-}, \theta\right), \\
& 0=\left(\sigma_{r \theta}^{p}+\sigma_{r \theta}^{s}\right)\left(a^{-}, \theta\right),
\end{aligned}
$$

for all $\theta$. From (B.16) and from the fields (B.13), (B.14), (B.15) and (B.15), we get an infinity of linear systems. For computational purpose, these systems are computed up to $N_{\text {Bessel }}$ terms, hence

$$
\boldsymbol{M}_{n} \boldsymbol{X}_{n}=\boldsymbol{Y}_{n}, \quad n=0,1, \ldots, N_{\text {Bessel }}
$$

with

$$
\boldsymbol{X}_{n}=\left(\begin{array}{c}
R_{n} \\
T_{n}^{p} \\
T_{n}^{s}
\end{array}\right), \quad \boldsymbol{Y}_{n}=\left(\begin{array}{c}
-\frac{1}{c_{1}} \varepsilon_{n} i^{n} J_{n}^{\prime}\left(-k_{1} a\right) A S \\
-\rho_{1} \varepsilon_{n} i^{n} J_{n}\left(-k_{1} a\right) A S \\
0
\end{array}\right)
$$


The coefficients of the matrices $\boldsymbol{M}_{n}$ are

$$
\begin{aligned}
& \boldsymbol{M}_{n}[1,1]=\frac{1}{c_{1}} H_{n}^{\prime}\left(-k_{1} a\right), \quad \boldsymbol{M}_{n}[1,2]=-\frac{1}{c_{p 2}} J_{n}^{\prime}\left(-k_{p 2} a\right), \\
& \boldsymbol{M}_{n}[1,3]=\frac{n}{a \omega} J_{n}\left(-k_{s 2} a\right), \quad \boldsymbol{M}_{n}[2,1]=\rho_{1} H_{n}\left(-k_{1}, a\right), \\
& \boldsymbol{M}_{n}[2,2]=\frac{1}{\omega^{2}}\left((\lambda+2 \mu) k_{p 2}^{2} J_{n}^{\prime \prime}\left(-k_{p 2} a\right)-\lambda \frac{k_{p 2}}{a} J_{n}^{\prime}\left(-k_{p 2} a\right)-\lambda\left(\frac{n}{a}\right)^{2} J_{n}\left(-k_{p 2} a\right)\right) \\
& \boldsymbol{M}_{n}[2,3]=-\frac{2 \mu}{\omega^{2}}\left(\frac{n}{a^{2}} J_{n}\left(-k_{s 2} a\right)+\frac{n k_{s 2}}{a} J_{n}^{\prime}\left(-k_{s 2} a\right)\right), \quad \boldsymbol{M}_{n}[3,1]=0 \\
& \boldsymbol{M}_{n}[3,2]=\frac{2}{\omega^{2}}\left(\frac{n}{a^{2}} J_{n}\left(-k_{p 2} a\right)+\frac{n k_{p 2}}{a} J_{n}^{\prime}\left(-k_{p 2} a\right)\right), \\
& \boldsymbol{M}_{n}[3,3]=-\frac{1}{\omega^{2}}\left(k_{s 2}^{2} J_{n}^{\prime \prime}\left(-k_{s 2} a\right)+\frac{k_{s 2}}{a} J_{n}^{\prime}\left(-k_{s 2} a\right)+\left(\frac{n}{a}\right)^{2} J_{n}\left(-k_{s 2} a\right)\right) .
\end{aligned}
$$

Step 5. We express the acoustic and elastic fields in cartesian coordinates. To do so, we use the well-known rotation formulas [8]

$$
\left(\begin{array}{c}
v_{1} \\
\sigma_{11} \\
\sigma_{12} \\
\sigma_{22}
\end{array}\right)=\left(\begin{array}{ccccc}
\cos \phi-\sin \phi & 0 & 0 & 0 \\
\sin \phi & \cos \phi & 0 & 0 & 0 \\
0 & 0 & \cos ^{2} \phi & -2 \sin \phi \cos \phi & \sin ^{2} \phi \\
0 & 0 & \sin \phi \cos \phi \cos ^{2} \phi-\sin ^{2} \phi-\sin \phi \cos \phi \\
0 & 0 & \sin ^{2} \phi & 2 \sin \phi \cos \phi & \cos ^{2} \phi
\end{array}\right)\left(\begin{array}{c}
v_{r} \\
\sigma_{r \theta} \\
v_{\theta \theta}
\end{array}\right) .
$$

Note that the scalar pressure $p$ is the same in cylindrical and in cartesian coordinates. 
Step 6. The last step, concerning the inverse discrete Fourier transform, is classical and is not detailed here.

Acknowledgments. We wish to thank J.P. Sessarego (LMA-CNRS, Marseille, France) who supported this research.

\section{References}

[1] K. Aki, P. G. Richards, Quantitative Seismology. Theory and Methods. Vol. 1, Freeman, New-York, 1980.

[2] L. M. Brekhovskikn, O. A. Godin, Acoustics of Layered Media 1-Plane and Quasi-Plane Waves, Springer-Verlag, Berlin Heidelberg, 1990.

[3] D. Calhoun, R. J. LeVeque, Solving the advection-diffusion equation in irregular geometries, J. Comput. Phys. 156 (2000) pp. 1-38.

[4] F. Collino, P. Joly, F. Millot, Fictitious domain method for unsteady problems: application to electromagnetic scattering, J. Comput. Phys., 138 (1997), pp. 907-938.

[5] R. Fedkiw, T. Aslam, B. Merriman, S. Osher, A non-oscillatory eulerian approach to interfaces in multimaterial flows: the Ghost-Fluid Method, J. Comput. Phys., 152 (1999), pp. 457-492.

[6] T. FogarTy, Finite Volume Methods for Acoustics and Elasto-plasticity with Damage in a Heterogeneous Medium, PhD. thesis, University of Washington, Seattle, WA, 2001.

[7] T. Fouquet, Raffinement de maillage spatio-temporel pour les équations de Maxwell, PhD. thesis, Paris 9, 2000.

[8] P. Germain, P. Muller, Introduction à la mécanique des milieux continus, Masson, 1995.

[9] E. Godlewski, P. A. Raviart, Numerical Approximation of Hyperbolic Systems of Conservation Laws, Springer-Verlag, 1996.

[10] D. Komatitsch, C. Barnes, J. Tromp, Wave propagation near a fluid-solid interface: a spectral-element approach, Geophysics, 65-2 (2000), pp. 623-631.

[11] L. LeE, Immersed Interface Methods, PhD. thesis, University of Washington, Seattle, WA, 2002.

[12] L. Lee, R. J. LeVeque, Immersed Interface Method for incompressible Navier-Stokes equations, submitted to SIAM J. Scient. Comput. (2002).

[13] R. J. LeVeque, Numerical Methods for Conservation Laws, Birkhauser, 1990. 
[14] R. J. LeVeque, Wave propagation algorithms for multi-dimensional hyperbolic systems, J. Comput. Phys., 131 (1997), pp. 327-353.

[15] Z. LI, M. C. LAI, The Immersed Interface Method for the Navier-Stokes equations with singular forces, J. Comput. Phys., 171-2 (2001), pp. 822-842.

[16] Z. LI, R. J. LeVeque, Immersed interface methods for Stokes flow with elastic boundaries or surface tension, SIAM J. Sci. Comput., 18 (1997), pp. 709-735.

[17] Z. LI, R. J. LeVEQue, The immersed interface method for elliptic equations with discontinuous coefficients and singular sources, SIAM J. Num. Anal., 31 (1994), pp. 1019-1044.

[18] B. Lombard, Modélisation Numérique de la Propagation des Ondes Acoustiques et Elastiques en Présence d'Interfaces, $\mathrm{PhD}$. thesis, University of Aix-Marseille II (2002).

[19] B. Lombard, J. Piraux, How to incorporate the spring-mass conditions in finite-difference schemes, SIAM J. Scient. Comput., 24 (2003), pp. 1379-1407.

[20] P. M. Morse, H. Feshbach, Methods of Theoretical Physics, McGraw-Hill Book Company (1953).

[21] C. S. Peskin, Numerical analysis of blood flow in the heart, J. Comput. Phys., 25 (1977), pp. 220-252.

[22] J. Piraux, B. Lombard, A new interface method for hyperbolic problems with discontinuous coefficients: one-dimensional acoustic example, J. Comput. Phys., 168-1 (2001), pp. 227-248.

[23] W. H. Press, S. A. Teukolskyn, W. T. Vetterling, B. P. Flannery, Numerical Recipes in Fortran: The Art of Scientific Computing, Cambridge University Press (1992).

[24] R. van Vossen, J. O. Robertsson, C. H. Chapman, Finite-difference modeling of wave-propagation in a fluid-solid configuration, Geophysics, 67-2 (2002), pp. 618-624.

[25] A. Wiegmann, K. Bube, The Immersed Interface Method for nonlinear differential equations with discontinuous coefficients and singular sources, SIAM J. Numer. Anal, 35 (1998), pp. 177-200.

[26] A. Wiegmann, K. Bube, The Explicit Jump Immersed Interface Method: finite-difference methods for PDE with piecewise smooth solutions, SIAM J. Numer. Anal, 37 (2000), pp. 827-862.

[27] C. Zhang, Immersed Interface Method for Hyperbolic Systems of Partial Differential Equations with Discontinuous Coefficients, PhD. thesis, University of Washington, Seattle, WA, 1996.

[28] C. Zhang, R. J. LeVeque, The Immersed Interface Method for acoustic wave equations with discontinuous coefficients, Wave Motion, 25 (1997), pp. 237263. 